\title{
Identification of HMMR as a prognostic biomarker for patients with lung adenocarcinoma via integrated bioinformatics analysis
}

Zhaodong $\mathbf{L i}$ Equal first author, 1 , Hongtian Fei ${ }^{\text {Equal first author, } 2}$, Siyu Lei ${ }^{1}$, Fengtong Hao ${ }^{1}$, Lijie Yang ${ }^{1}$, Wanze $\mathbf{L i}^{1}$, Laney Zhang

${ }^{3}$, Rui Fei ${ }^{\text {Corresp. } 1,4}$

${ }^{1}$ Department of Cell Biology, College of Basic Medical Sciences, Jilin University, Changchun, Jilin, China

2 Department of Pharmacology, College of Basic Medical Sciences, Jilin University, Changchun, Jilin, China

3 The College of Arts and Sciences, Cornell University, New York, USA

4 Key laboratory of Iymphatic surgery Jilin province, Jilin University, Changchun, Jilin, China

Corresponding Author: Rui Fei

Email address: feirui@jlu.edu.cn

Background: Lung adenocarcinoma (LUAD) is the most prevalent tumor in lung carcinoma cases and threatens human life seriously worldwide. Here we attempt to identify a prognostic biomarker and potential therapeutic target for LUAD patients.

Methods: Differentially expressed genes (DEGs) shared by GSE18842, GSE75037, GSE101929 and GSE19188 profiles were determined and used for protein-protein interaction analysis, enrichment analysis and clinical correlation analysis to search for the core gene, whose expression was further validated in multiple databases and LUAD cells (A549 and PC-9) by quantitative real-time PCR (qRT-PCR) and western blot analyses. Its prognostic value was estimated using the Kaplan-Meier method, meta-analysis and Cox regression analysis based on the Cancer Genome Atlas (TCGA) dataset and co-expression analysis was conducted using the Oncomine database. Gene Set Enrichment Analysis (GSEA) was performed to illuminate the potential functions of the core gene. Results: A total of 115 shared DEGs were found, of which 24 DEGs were identified as candidate hub genes with potential functions associated with cell cycle and FOXM1 transcription factor network. Among these candidates, HMMR was identified as the core gene, which was highly expressed in LUAD as verified by multiple datasets and cell samples. Besides, high HMMR expression was found to independently predict poor survival in patients with LUAD. Co-expression analysis showed that HMMR was closely related to FOXM1 and was mainly involved in cell cycle as suggested by GSEA. Conclusion: HMMR might be served as an independent prognostic biomarker for LUAD patients, which needs further validation in subsequent studies. 
1 Identification of HMMRas a prognosticbiomarker for

2 patients with lung adenocarcinoma via integrated

3 bioinformatics analysis

4 Zhaodong Li ${ }^{1 \dagger}$, Hongtian Fei ${ }^{\dagger \dagger}$, Siyu Lei ${ }^{1}$, Fengtong Hao' ${ }^{1}$, Lijie Yang', Wanze Li ${ }^{1}$,

5 Laney Zhang ${ }^{3}$, Rui Fei, ${ }^{1,4}$,

6 1Department of Cell Biology, College of Basic Medical Sciences, Jilin University, Changchun,

7 Jilin, China

$8{ }^{2}$ Department of Pharmacology, College of Basic Medical Sciences, Jilin University, Changchun,

9 Jilin, China

$10{ }^{3}$ The College of Arts and Sciences, Cornell University, Ithaca, New York, USA

$11{ }^{4}$ Key laboratory of lymphatic surgery Jilin province, Jilin University, Changchun, Jilin, China

\section{"Corresponding Author:}

Rui Fei

Department of Cell Biology, College of Basic Medical Sciences, Jilin University, Changchun, Jilin 130000, China

Email address: feirui@jlu.edu.cn

†These authors have contributed equally to this work and share first authorship.

\section{Abstract}

Background: Lung adenocarcinoma (LUAD) is the most prevalent tumor in lung carcinoma cases and threatens human life seriously worldwide. Here we attempt to identify a prognostic biomarker and potential therapeutic target for LUAD patients.

Methods: Differentially expressed genes (DEGs) shared byGSE18842, GSE75037, GSE101929 and GSE19188 profiles were determined and used for protein-protein interaction analysis, enrichment analysis and clinical correlation analysis to search for the core gene, whose expression was further validated in multiple databases and LUAD cells (A549 and PC-9) by quantitative real-time PCR (qRT-PCR) and western blot analyses. Its prognostic value was estimated using the Kaplan-Meier method, meta-analysis and Cox regression analysis based on the Cancer Genome Atlas (TCGA) dataset and co-expression analysis was conducted using the Oncomine database. Gene Set Enrichment Analysis (GSEA) was performed to illuminate the potential functions of the core gene.

Results: A total of 115 shared DEGs were found, of which 24 DEGs were identified as candidate hub genes with potential functions associated with cell cycle and FOXM1 transcription factor network. Among these candidates, $H M M R$ was identified as the core gene, which was 
34 highly expressed in LUAD as verified by multiple datasets and cell samples. Besides, high

$H M M R$ expression was found to independently predict poor survival in patients with LUAD. Coexpression analysis showed that HMMR was closely related to FOXMland was mainly involved in cell cycle as suggested by GSEA.

Conclusion: $H M M R$ might be served as an independent prognostic biomarker for LUAD patients, which needs further validation in subsequent studies.

\section{Keywords: Lung adenocarcinoma, prognosis, $H M M R$, bioinformatics, differentially expressed genes}

\section{INTRODUCTION}

Lung cancer remains the most common cause of cancer-related death worldwide. More than $1,600,000$ patients are newly diagnosed with lung cancer each year, which reduces patients' life quality and brings heavy financial burden to the patients (Qu et al., 2020). Lung adenocarcinoma (LUAD) is the most frequent histological type among lung cancers, which occupies approximately 40\% (Bai et al., 2019). Although the clinical strategies treating LUAD, such as chemotherapy, radiotherapy, targeted therapy, surgery, and immunotherapy, have been developed in recent decades, the five-year survival rate of LUAD remains unsatisfactory, mainly because of the lack of effective prognostic biomarkers and therapeutic targets involved in the development and progression of the lung carcinoma (Chen et al., 2019). It is therefore crucial to perform an integrated study to identify the core genes and comprehensively understand its relevant signaling pathways during the LUAD occurrence and progression.

Nowadays, the combination of genomics technology and bioinformatics analysis facilitated the wide application of gene expression profiles in a range of human cancers, providing a new insight into screening tumor-associated genes and identifying the core prognosis factors (Kaushik, et al., 2019; Huang et al., 2019; Liu et al., 2019). Meanwhile, high-throughput technologies simplified the procedure of gene expression profiling and a growing body of expression data can be retrieved from public databases, which allows us to further study the underlying molecular mechanisms and medicine targets in LUAD (Guo et al., 2020; Sun et al., 2020). Although many studies have identified hundreds of differentially expressed genes (DEGs) and indicated corresponding biological pathways associated with lung cancer, the results were not consistent because of various reasons (Long et al., 2020; Mao et al., 2019; Lu et al., 2020). Considering the limitation of a single microarray analysis, characterized by limited samples, unbalanced datasets and serious systematic error, we herein conducted an integration analysis of genetic data with multiple gene expression profiles and public databases to overcome the shortage and obtain reliable diagnosis markers.

In this work, multiple gene expression profiles retrieved from the Gene Expression Omnibus (GEO) database (https://www.ncbi.nlm.nih.gov/geo/) were used for identification of DEGs and candidate core genes. Protein-protein interaction (PPI) was then constructed following the identification and validation of the hub gene. In addition, survival analysis, meta-analysis, prognostic analysis, and co-expression analysis were performed successively to evaluate the 
potential of the core gene as a prognostic factor in LUAD. The biological functions of the hub gene were explored through the Gene Set Enrichment Analysis (GSEA) in our study. The workflow of this work is provided in Figure 1.

\section{MATERIALS AND METHODS}

\section{Data collection and processing}

Eight gene expression profiles were obtained from the GEO online public database, which was provided in Supplementary Table S1. The adjusted $p$-value $<0.05$ and $\mid \log _{2}$ (foldchange) $\mid \geq$ 2 were set as cut-off values for screening DEGs in GSE18842, GSE19188, GSE75037 and GSE101929 profiles using R package "limma". Subsequently, the common DEGs shared by the four profiles were used for further study. Additionally, transcriptome RNA-sequencing and proteomics data were extracted from the Cancer Genome Atlas (TCGA) database (https://cancergenome.nih.gov/) andthe Clinical Proteomic Tumor Analysis Consortium (CPTAC) database (https://cptac-data-portal.georgetown.edu/), repectively.

\section{PPI network construction and enrichment analysis}

Construction of PPI network and identification of key module were constructed as previously described in Li et al. (2021). Then, the genes with the highest connectivity degrees in key module were recognized as candidate hub genes, whose mRNA expression levels were further validated via GSE19804 and TCGA datasets. The FunRich software (version 3.1.3) was applied to perform enrichment analysis in biological process, cellular component, molecullar function and biological pathway with the cutoff criteria of $p<0.05$ for the corresponding genes in key module.

\section{Hub gene identification and validation}

Correlations between candidate hub genes and tumor stages were determined by Pearson's correlation analysis in GSE31210 profile and TCGA dataset using R package "corrplot". Then, the common genes shared by the two datasets were identified as hub genes. Furthermore, the protein expression levels of hub geneswere further verified utilizing CPTAC data and immunohistochemical results from Human Protein Atlas (HPA) (https://www.proteinatlas.org). In addition to public datasets, we performed qRT-PCR and western blotting to measure the expression pattern of the core gene in LUAD cells (A549 and PC-9).

\section{Survival analysis and prognostic value analysis}

The association of hub genes with overall survival (OS), progress-free survival (PFS) and disease-free survival (DFS) in LUAD patients were conducted as previously described in Li et al. (2021). Meanwhile, Kaplan-Meier Plotter (www.kmplot.com) and Gene Expression Profiling Interactive Analysis (GEPIA) (http://gepia.cancer-pku.cn/) platforms were also used to perform 
107

108

109

110

111

112

113

114

115

116

117

118

119

120

121

survival analysis for the corresponding genes. Additionally, the prognostic estimation of hub genes using meta-analysis was performed based on the methods in Li et al. (2021). Finally, Cox regression analysis was conducted following the protocols in Li et al. (2020).

\section{Co-expression analysis and gene set enrichment analysis (GSEA)}

Co-expression analyses for hub genes were conducted using the Oncomine database (https://www.oncomine.org) in order to identify a significant factor associated with hub genes, which was further verified in both GSE31210 profile and TCGA dataset using R package "corrplot" and GraphPad Prism software (verson 7.0). Additionally, GSEA software (version 4.0.3) was utilized to conduct enrichment analysis for DEGs between high and low hub gene expression subgroups to investigate the biological functions of hub gene. FDR $<0.05$ and $p<0.05$ were set as the cut-off criteria.

\section{Cell culture}

HBE, A549 and PC-9 cells were cultured according to protocols in Li et al. (2021).

\section{Quantitative RT-PCR analysis}

Quantitative RT-PCR analysis was conducted following the instructions in Li et al. (2021). The primer sequences are as follows: $H M M R$ forward, 5'-ATGATGGCTAAGCAAGAAGGC3' and reverse, 5'-TTTCCCTTGAGACTCTTCGAGA-3'; GAPDH forward, 5'GGAGCGAGATCCCTCCAAAAT-3' and reverse, 5'- GGCTGTTGTCATACTTCTCATGG-3'.

\section{Western blot analysis}

Westerm blot analysis was subsequently performed according to protocols in Li et al. (2021). The primary antibody against HMMR was purchased from Abcam (Cambridge, UK, diluted 1:1000, ab124729).Statistical analysis

The R (version 3.6.0) and Graphpad prism software (version 7.0) were used to perform statistical analysis. Cochran's Q test and Higgin's $I^{2}$ statistics were applied for the heterogeneity estimation in the meta-analysis in meta-analysis. The statistical differences in the Kaplan-Meier analysis were calculated by log-rank test. Besides, data are presented as the mean $\pm \mathrm{SD}$ of at least three independent experiments. The $2^{-\Delta \Delta \mathrm{Ct}}$ method was utilized to analyze the results of qRT-PCR and Student's t-test was applied to assess the significance of differences between groups. A $p$-value of $<0.05$ was considered statistically significant.

\section{RESULTS}

\section{Identification of DEGs in LUAD}

A total of 429, 287, 672, 513 DEGs were identified in GSE18842, GSE19188, GSE75037 
and GSE101929, with 194, 105, 223, 174 up-regulated genes and 235, 182, 449, 339 downregulated genes, respectively (Figures 2A-D). Among them, 115 DEGs were shared by the four datasets, including 38 up-regulated genes and 77 down-regulated genes (Figures 2E, F and Supplementary Table S2).

\section{Construction of PPI network and module analysis}

The shared DEGs were used to construct the PPI network, which included 87 nodes and 399 edges (Figure 3A). We also identified a key module (cluster 1) with the highest MCODE score (20.92) from the PPI network, which contained 24 genes. Meanwhile, supplementary Table S2 provided more details for the 24 genes. Afterwards, these 24 genes were significantly associated with Cell Cycle, Spindle assembly, Cell growth and/or maintenance, Cell communication and Signal transduction (Figure 3B and Supplementary Table S3). Centrosome, Microtubule, Spindle microtubule and other cellular components were mainly enriched by the 24 DEGs (Supplementary Figure 1A and Supplementary Table S4). Besides, the corresponding DEGs mainly enriched in Motor activity, Kinase binding, Protein serine/threonine kinase activity and other molecullar functions (Supplementary Figure 1B and Supplementary Table S5). Meanwhile, Kinesins, Cell Cycle, FOXM1 transcription factor network were the main enriched biological pathways for these 24 DEGs (Figure 3C and Supplementary Table S6).

\section{Identification of HMMR as a hub gene in LUAD}

Besides, among the 24 DEGs with high connectivity degrees in the whole PPI network (Figure 3A and Figure 4A), 19 DEGs were identified with leading intramodular connectivity according to the key module (cluster 1) PPI network (pink nodes constructing the network in Figure 3A) and meanwhile, they were considered as the candidate hub genesin the work (Figure 4B) . Afterwards, the candidate hub genes were identified in the GSE19804 and GSE31210 profiles (Figures 4C and D). In addition to the difference analysis, the GSE31210 profile and the TCGA dataset were used to evaluate the correlation between the expression of the candidate hub genes and tumor stages (Table 1). The results revealed that $H M M R$ was uniquely shared by these two datasets among the top three genes most strongly correlated with tumor stages (Figure 5A). Though $p$-values were significant, $\mathrm{R}$ values in correlation analyses of $H M M R$ expression and tumor stage were not satisfied. Therefore, we further performed differential analysis for $H M M R$ expression in LUAD patients with different tumor stage aiming to supply the unsatisfied correlation. In the work, $H M M R$ was considered as the hub gene. It was highly expressed in LUAD tissues compared with normal lung tissues according to non-paired (Figure 5D) and paired (Figure 5E) statiscal analysis in TCGA dataset. Similarly, the protein expression level of HMMR was signifcantly up-regulated in LUAD samples versus normal samples based on CPTAC (Figure 5F) and HPA (Figure 5G) datasets. Furthermore, the results were further validated using qRT-PCR and western blot analyses ((Figures $\mathbf{5 H}$ and I).

\section{$H M M R$ served as a prognostic factor in LUAD}


176

177

178

179

180

181

182

183

It was found that high $H M M R$ expression significantly deteriorated overall survival (OS) in TCGA dataset, GSE68465 profile, GSE50081 profile, Kaplan-Meier Plotter and GEPIA platforms (Figures 6A-E), as presented in GSE31210 profile (Supplementary Figure 2). Besides, HMMR was negatively associated with progression-free survival (PFS) in TCGA dataset, GSE68465 profile, Kaplan-Meier Plotter platform (Figures 6F-H). The associations of $H M M R$ with inferior disease-free survival (DFS) were also observed in Figures 6I and $\mathrm{J}$ using GSE50081 profile and GEPIA platform. Because of the unsignificant heterogeneity $\left(I^{2}<50 \%\right.$, $p>0.05$ ), we selected solid model to perform the meta-analysis. The results of the meta-analysis implied that HMMR served as a prognostic factor for the OS of LUAD patients (Figure 6K). Moreover, the Cox regression analysis revealed that $H M M R$ acted as an independent prognostic factor for the survival of LUAD patients (Table 2).

\section{Prognostic value of $H M M R$ in LUAD}

The HMMR expression was significantly impacted by gender (female $v s$. male), tumor stage (stage I\&II vs. stage III\&IV), T classification (T1 vs. T2-4), N classification (N0 vs. N1-3), and $\mathrm{M}$ classification (M0 vs. M1), whereas not by age (<=65 vs. $>65)$ (Figure 7A). The LUAD patients were classified into high and low $H M M R$ expression subgroups according to the median of $H M M R$ expression levels. Subsequent survival analysis showed that high $H M M R$ expression group exhibited significantly poor OS in $>65$ age, female, male, stage III\&IV, M0 and N1-3 subgroups (Figure 7B). Interestingly, there was no statistical significance for HMMR expression between $>65$ age subgroup and $<=65$ age subgroup, while expression level of $H M M R$ significantly impacted the OS for LUAD patients with above 65 age. To some extent, there was contradictory for the results might due to limited samples and unbalanced data, which was needed further investigation.

\section{HMMR co-expressed with FOXM1 in LUAD}

According the results of pathway enrichment analysis, we have identified the FOXMI transcription factor network and cell cycle were the main biological pathway enriched by the common 24 DEGs (Figure 3C). Among the pathways, FOXM1 (forkhead box protein M1) was an essential proliferation-associated transcription factor. It was widely expressed during cell cycle and involved in cellular growth, self-renewal, and tumorigenesis (Liao et al., 2018). Additionally, through Oncomine co-expression analysis, we found that the expression of $H M M R$ was positively associated with that of FOXM1 (r=0.890) (Figure 8A), which was consistent with our expectation. The mRNA expression of FOXM1 exhibited significant elevation in LUAD tissues relative to the matched normal lung tissues in both the GSE31210 profile and the TCGA data. In line with Oncomine database, the GSE31210 profile and the TCGA data also suggested that the expressions of $H M M R$ and FOXMIwere positively correlated via Pearson's correlation analysis, even with similar correlation strengths, with $\mathrm{r}=0.723, p<0.001$ and $\mathrm{r}=0.730, p<0.001$, respectively (Figures $\mathbf{8 B}, \mathbf{C}$ ). 
213

214

215

216

217

218

219

220

221

222

223

224

225

226

227

228

229

230

231

232

233

234

235

236

237

238

239

240

241

242

243

244

245

246

247

248

249

250

251

\section{HMMR was involved in cell cycle in LUAD}

GSEA was performed to illuminate the potential biological functions of $H M M R$ in LUAD progression using DEGs between high and low $H M M R$ subgroups according to the median $H M M R$ expression level. As shown in Figure 9A and Supplementary Table S7, the functions of these genes were significantly associated with cell cycle, G2/M phase transition, cell cycle process, chromosome segregation, and nuclear division pathways, as indicated by biological process enrichment analysis. DNA repair, E2F targets and G2/M checkpoint were the major enriched HALLMARK terms (Figure 9B and Supplementary Table S8). Cell cycle, DNA replication, p53 signaling pathway, and other biological pathways were the major enriched HALLMARK terms (Figure 9C and Supplementary Table S9). Taken together, those results indicated that the implication of HMMR in LUAD progression might be mediated by cell cycle regulation.

\section{DISCUSSION}

Currently, LUAD is considered to be the most common subtype of lung cancer in clinical practice, with a five-year overall survival rate varying from $4 \%$ to $17 \%$ (Hirsch et al., 2017). The exact pathogenesis of LUAD remains elusive and numerous diverse and complicated processes have been reported to play a role. For instance, the presence of aberrant gene expression, autophagy activation, unexpected tumor microenvironment, immune cell infiltration, abnormal cell cycle, DNA methylation, epigenetic interactions, and other molecular and cellular events, have been identified to be associated with the development of LUAD (Fan et al., 2019; Chen et al., 2019; Wang et al., 2019; Lazarus et al., 2018). It has also been reported that several signaling pathways, such as MAPK pathway, AKT-mTOR signaling and $\mathrm{Wnt} / \beta$-catenin signaling pathway, are frequently activated in LUAD (Hou et al., 2019; Liu et al., 2020; Luo et al., 2019). In spite of the great progress in illustrating the pathogenesis of LUAD and treating LUAD, effective prognostic biomarkers and treatment targets are stilling lacking. Hence, there is an urgent need to find a precise and effective prognostic factor and a therapeutic target in order to improve LUAD-associated survival rate.

Bioinformatics is a data-driven subtype of science and currently frequently applied in aspects like analyzing genetic data, studying tumor progression, screening core genes, and identifying the medical targets through the usage of bioinformatic analytical tools including software plugins and packages, as well as database platforms (Luo et al., 2019; Li et al., 2019). Previously, an integrated regulatory network, containing DEGs, transcription factors, and miRNAs, has been constructed using GSE37764 dataset, which attempted to find out the important factors in the pathogenesis of lung adenocarcinoma and lay the foundation for the further investigation ( $D u$ et al., 2015). Meanwhile, an eight-gene prognostic signature (DLGAP5, KIF11, RAD51AP1, $C C N B 1, A U R K A, C D C 6$, OIP5 and NCAPG) also has been identified for the survival of LUAD using GSE19188 and GSE33532 profiles ( $L i$ et al., 2018).Besides, Zhou et al. aimed to screen out the driver genes in smoking-associating lung adenocarcinoma via bioinformatics, and then they suggested that the seven genes (CYP17A1, PKHD1L1, RPE65, NTSR1, FETUB, IGFBP1 
252

253

254

255

256

257

258

259

260

261

262

263

264

265

266

267

268

269

270

271

272

273

274

275

276

277

278

279

280

281

282

283

284

285

286

287

288

289

290

291

292

and $G 6 P C$ ) could be promising prognostic factors for lung adenocarcinoma according to their negative correlation with the patient survival (Zhou et al., 2019).

In this study, we aimed to find a core gene that has both prognostic value and the potential to become a treatment target for LUAD via integrated analysis. Firstly, shared DEGs by the four GEO profiles were obtained and used for PPI network construction followed by key module identification in order to identify candidate hub genes. Secondly, biological process and pathway enrichment analyses for the identified genes were carried out by the FunRich software. The enrichment results suggested that the potential functions of those candidate genes were specifically associated with cell cycle, spindle assembly, FOXMI transcription factor network, $\mathrm{M}$ phase, and other pathways. It is well known that cell cycle and $M$ phase transition play essential roles in manipulating the proliferation of cells and the occurrence of tumors. For example, repressing p21 expression, the check point of cell cycle, could promote tumor proliferative capacity and accelerate cancer procession (Zhang et al., 2019). Likewise, many molecules, like Rac3, SMAD3, CDCA7 and DMBX1, which regulate cell growth via cell cycle, have similar functions (Li et al., 2017; Wang et al., 2019; Luo et al., 2019). Accumulating studies have reported the involvement of FOXM1 in the progression of tumors including lung cancer, gastric cancer, breast cancer, and epithelial ovarian cancer (Hsieh et al., 2019; Bai et al., 2019; Ring et al., 2018; Wang et al., 2016).Specifically, in lung cancer, FOXM1 has been found to be coexpressed with CENE and could regulate the expression of $M M P 2$, contributing to LUAD growth and metastasis (Shan et al., 2019; Hsieh et al., 2019). Besides, supporting our findings in the present study, FOXMI transcription factor network was identified as a major predictor of poor outcomes in pan-cancer in the report of Andrew J Gentles et al. (Gentles et al.,2015). Finally, among these candidate genes, $H M M R$ was identified as the hub gene in this study due to its significant role in predicting survival and its association with tumor stage, which was further validated at the protein level in the HPA database and at the mRNA expression level on the CCLE platform. The potential functions of the hub gene were explored by GSEA, and the results indicated that mainly cell cycle and its relevant pathways were activated pathways in LUAD highly expressing $H M M R$.

The above results confirmed the prognostic value of HMMR expression in LUAD. HMMR, a sulfonated glycosaminoglycan, is a receptor for hyaluronic acid (HA), which accumulated during pulmonary inflammation. It has been found that high expression of HMMR is associated with multiple human malignancies, such as gastric cancer, breast cancer, prostate cancer, ovarian cancer, bladder cancer, with characteristics of promoting cancer progression and indicating poor prognosis in patients (Huang et al., 2017; Yeh et al., 2018; Rizzardi et al., 2017). For example, a zebrafish xenograft assay verified that highly expressed $H M M R$, under the control of both TGF $\beta$ signaling and Hippo pathway, contributed to sarcoma genesis and metastasis (Ye et al., 2020). In gastric cancer patients, $H M M R$ over-production was remarkably associated with tumor relapse and poor prognosis, and resulted in resistance to the chemotherapy via promoting epithelialmesenchymal transition and modulating cancer stem cell properties (Zhang et al., 2019). Additionally, HMMR also interacted with CD44, another HA receptor characterized by forming complexes with ERK1/2, to exert its functions in breast cancer (Telmer et al., 2011).Besides, 
293

294

295

296

297

298

299

300

301

302

303

304

305

306

307

308

309

310

311

312

313

314

315

316

317

318

319

320

321

322

323

324

325

326

327

328

HMMR was identified as a promising diagnostic biomarker and an independent prognostic factor for hepatocellular carcinoma (HCC) via bioinformatics, the expression of which was positively correlated with HCC tumor grade and stage in HCC patients (Lu et al., 2020).

We found that high expression of $H M M R$ was significantly correlated with poorer OS and survival rate in subgroups with clinical stage III/IV, lymph node metastasis classification $1 / 2 / 3$, male, female and patients with an age of above 65 . Multivariate COX regression analysis further suggested that the HMMR expression level could serve as an independent prognostic indicator in LUAD. These findings highlighted the prognostic value of HMMR expression in LUAD. We further conducted correlation analysis based on the Oncomine database and the results indicated the expression of $H M M R$ was positively associated with that of FOXM1, which encoded transcription factor of fork head family and intriguingly was also found to negatively impact prognosis in many solid tumors (Liang et al., 2019; Liu et al., 2018). Additionally, cell cycle, DNA replication, p53 signaling pathway, RNA degradation, spliceosome, and ubiquitin mediated proteolysis were significantly enriched pathways in LUAD highly expressing $H M M R$. It was well known that these six signaling pathways were typically involved in the occurrence and development of cancers (Aubrey et al., 2018; Fish et al., 2019; Dvinge et al., 2019; Senft et al., 2018). For example, the core protein and RNA components of the spliceosome were essential for splicing decision and able to manipulate the splice sites during pre-mRNA processing, thereby playing importantly regulatory roles in tumorigenesis and metastasis of malignant cells ( $\mathrm{Hsu}$ et al., 2015). However, there was no inhibitor of HMMR currently undergoing preclinical or clinical testing anywhere in the world.

In the work, we identified $H M M R$ as the core gene via integrated bioinformatics analysis and provided robust evidence for the potential prognostic and therapeutic role of $H M M R$ in LUAD, though the prognostic value was not significant in patients with clinical stage I/II and an age of below 65. It might also be a limitation, as only RNA-based bioinformatics analysis was performed in this study and no functional experiment was conducted. Therefore, further studies are needed to improve the reliability of the results.

\section{CONCLUSION}

In summary, $H M M R$ might serve as an independent prognostic factor for the OS in LUAD patients. This work would facilitate the development of novel prognostic biomarkers and therapy targets for LUAD, though further experiments are needed to verify these findings.

\section{ACKNOWLEDGEMENT}

We would like to thank TopEdit (www.topeditsci.com) for its linguistic assistance during the preparation of this manuscript. Besides, we are extremely grateful for reviewers' input in helping this manuscript.

\section{Funding}


329 The authors received no funding for this work.

330 Competing Interests

331 The authors declare that they have no competing interests.

332 Author Contributions

333

334

335

336

337

338

339

340

341

342

343

344

345

346

347

348

349

350

351

352

353

354

355

356

357

358

359

360

361

362

363

- Zhaodong Li conceived and designed the experiments, performed the experiments, analyzed the data, prepared figures and/or tables, and approved the final draft.

- Hongtian Fei conceived and designed the experiments, performed the experiments, analyzed the data, prepared figures and/or tables, and approved the final draft.

- Siyu Lei performed the experiments, analyzed the data, prepared figures and/or tables, and approved the final draft.

- Fengtong Hao performed the experiments, analyzed the data, prepared figures and/or tables, and approved the final draft.

- Lijie Yang performed the experiments, analyzed the data, prepared figures and/or tables, and approved the final draft.

- Wanze Li performed the experiments, analyzed the data, prepared figures and/or tables, and approved the final draft.

- Laney Zhang analyzed the data, prepared figures and/or tables, and approved the final draft.

- Rui Fei conceived and designed the experiments, performed the experiments, analyzed the data, authored or reviewed drafts of the paper, and approved the final draft.

\section{Data Availability}

Publicly available datasets were analyzed in this study. This datacan be found at Gene Expression Omnibusdatabase (https://www.ncbi.nlm.nih.gov/geo/)andThe Cancer Genome Atlas database (https://cancergenome.nih.gov/)

\section{REFERENCES}

Aubrey BJ, Kelly GL, Janic A, Herold MJ, Strasser A.2018. How does p53 induce apoptosis and how does this relate to p53-mediated tumour suppression? Cell Death and Differentiation25(1):104-113.DOI 10.1038/cdd.2017.169.

Bai C, Liu X, Qiu C, Zheng J. 2019. FoxM1 is regulated by both HIF-1 $\alpha$ and HIF-2 $\alpha$ and contributes to gastrointestinal stromal tumor progression. Gastric Cancer22(1):91-103.DOI 10.1007/s10120-018-0846-6.

Bai Y, Xiong L, Zhu M, Yang Z, Zhao J, Tang H. 2019. Co-expression network analysis identified KIF2C in association with progression and prognosis in lung adenocarcinoma. Cancer Biomarkers24(3):371-382.DOI 10.3233/CBM-181512.

Chen D, Wang R, Yu C, Cao F, Zhang X, Yan F, Chen L, Zhu H, Yu Z, Feng J. 2019. FOXA1 contributes to acquisition of chemoresistance in human lung adenocarcinoma via 
transactivation of SOX5. EBioMedicine44:150-161. DOI 10.1016/j.ebiom.2019.05.046.

Chen Y, Chen H, Mao B, Zhou Y, Shi X, Tang L, Jiang H, Wang G, Zhuang W. 2019. Transcriptional Characterization Of The Tumor Immune Microenvironment And Its Prognostic Value For Locally Advanced Lung Adenocarcinoma In A Chinese Population. Cancer Management and Research 11:9165-9173. DOI 10.2147/CMAR.S209571.

Du J, Zhang L. 2015. Integrated analysis of DNA methylation and microRNA regulation of the lung adenocarcinoma transcriptome. ONCOLOGY REPORTS 34(2):585-594. DOI 10.3892/or.2015.4023.

Dvinge H, Guenthoer J, Porter PL, Bradley RK. 2019. RNA components of the spliceosome regulate tissue- and cancer-specific alternative splicing. Genome Research 29(10):15911604. DOI 10.1101/gr.246678.118.

Fan J, Zhang X, Wang S, Chen W, Li Y, Zeng X, Wang Y, Luan J, Li L, Wang Z, Sun X, Shen B, Ju D. 2019. Regulating autophagy facilitated therapeutic efficacy of the sonic Hedgehog pathway inhibition on lung adenocarcinoma through GLI2 suppression and ROS production. Cell Death\& Disease10(9):626. DOI 10.1038/s41419-019-1840-6.

Fish L, Navickas A, Culbertson B, Xu Y, Nguyen HCB, Zhang S, Hochman M, Okimoto R, Dill BD, Molina H, Najafabadi HS, Alarcón C, Ruggero D, Goodarzi H. 2019. Nuclear TARBP2 Drives Oncogenic Dysregulation of RNA Splicing and Decay. Molecular Cell75(5):967-981.e9. DOI 10.1016/j.molcel.2019.06.001.

Gentles AJ, Newman AM, Liu CL, Bratman SV, Feng W, Kim D, Nair VS, Xu Y, Khuong A, Hoang CD, Diehn M, West RB, Plevritis SK, Alizadeh AA. 2015. The prognostic landscape of genes and infiltrating immune cells across human cancers. Nature Medicine21(8):938-945. DOI 10.1038/nm.3909.

Guo Q, Ke XX, Liu Z, Gao WL, Fang SX, Chen C, Song YX, Han H, Lu HL, Xu G. 2020. Evaluation of the Prognostic Value of STEAP1 in Lung Adenocarcinoma and Insights Into Its Potential Molecular Pathways via Bioinformatic Analysis. Frontiers in Genetics11:242. DOI 10.3389/fgene.2020.00242.

Hirsch FR, Scagliotti GV, Mulshine JL, Kwon R, Curran WJ Jr, Wu YL, Paz-Ares L. 2017. Lung cancer: current therapies and new targeted treatments. Lancet389(10066):299-311. DOI 10.1016/S0140-6736(16)30958-8.

Hou XM, Zhang T, Da Z, Wu XA. 2019. CHPF promotes lung adenocarcinoma proliferation and anti-apoptosis via the MAPK pathway. Pathology Research and Practice215(5):988994. DOI 10.1016/j.prp.2019.02.005.

Hsieh NT, Huang CY, Li CC, Wang IC, Lee MF. 2019. MED28 and forkhead box M1 (FOXM1) mediate matrix metalloproteinase 2 (MMP2)-dependent cellular migration in human nonsmall cell lung cancer (NSCLC) cells. Journal of Cellular Physiology234(7):11265-11275. DOI 10.1002/jcp.27784.

Hsu TY, Simon LM, Neill NJ, Marcotte R, Sayad A, Bland CS, Echeverria GV, Sun T, Kurley SJ, Tyagi S, Karlin KL, Dominguez-Vidaña R, Hartman JD, Renwick A, Scorsone K, Bernardi RJ, Skinner SO, Jain A, Orellana M, Lagisetti C, Golding I, Jung SY, Neilson JR, Zhang XH, Cooper TA, Webb TR, Neel BG, Shaw CA, 
405

406

407

408

409

410

411

412

413

414

415

416

417

418

419

420

421

422

423

424

425

426

427

428

429

430

431

432

433

434

435

436

437

438

439

440

441

442

443

444

445

Westbrook TF. 2015. The spliceosome is a therapeutic vulnerability in MYC-driven cancer. Nature525(7569):384-388. DOI 10.1038/nature 14985.

Huang H, Huang Q, Tang T, Zhou X, Gu L, Lu X, Liu F. 2019. Differentially Expressed Gene Screening, Biological Function Enrichment, and Correlation with Prognosis in NonSmall Cell Lung Cancer. Medical Science Monitor25:4333-4341. DOI 10.12659/MSM.916962.

Huang M, Ma X, Shi H, Hu L, Fan Z, Pang L, Zhu F, Yang X, Xu W, Liu B, Zhu Z, Li C. 2017. FAM83D, a microtubule-associated protein, promotes tumor growth and progression of human gastric cancer. Oncotarget8(43):74479-74493. DOI 10.18632/oncotarget.20157.

Kaushik AC, Mehmood A, Wei DQ, Dai X. 2020. Systems Biology Integration and Screening of Reliable Prognostic Markers to Create Synergies in the Control of Lung Cancer Patients. Frontiers in Molecular Biosciences 7:47. DOI 10.3389/fmolb.2020.00047.

Lazarus KA, Hadi F, Zambon E, Bach K, Santolla MF, Watson JK, Correia LL, Das M, Ugur R, Pensa S, Becker L, Campos LS, Ladds G, Liu P, Evan GI, McCaughan FM, Le Quesne J, Lee JH, Calado D, Khaled WT. 2018. BCL11A interacts with SOX2 to control the expression of epigenetic regulators in lung squamous carcinoma. Nature Communications9(1):3327. DOI 10.1038/s41467-018-05790-5.

Li MY, Liu JQ, Chen DP, Li ZY, Qi B, He L, Yu Y, Yin WJ, Wang MY, Lin L. 2017. Radiotherapy induces cell cycle arrest and cell apoptosis in nasopharyngeal carcinoma via the ATM and Smad pathways. Cancer Biology \& Therapy18(9):681-693. DOI 10.1080/15384047.2017.

Li Q, Zhang LY, Wu S, Huang C, Liu J, Wang P, Cao Y. 2019. Bioinformatics Analysis Identifies MicroRNAs and Target Genes Associated with Prognosis in Patients with Melanoma. Medical Science Monitor25:7784-7794. DOI 10.12659/MSM.917082.

Li S, Xuan Y, Gao B, Sun X, Miao S, Lu T, Wang Y, Jiao W. 2018. Identification of an eightgene prognostic signature for lung adenocarcinoma. Cancer Management and Research 10:3383-3392. DOI 10.2147/CMAR.S173941.

Li Z, Qi F, Li F. 2020. Establishment of a Gene Signature to Predict Prognosis for Patients with Lung Adenocarcinoma. International Journal of Molecular Sciences 21(22):8479. DOI $10.3390 / \mathrm{ijms} 21228479$.

Li Z, Yu B, Qi F, Li F. 2021. KIF11 Serves as an Independent Prognostic Factor and Therapeutic Target for Patients With Lung Adenocarcinoma. Frontiers Oncology 11:670218. DOI 10.3389/fonc.2021.670218.

Liang J, Liu Z, Wei X, Zhou L, Tang Y, Zhou C, Wu K, Zhang F, Zhang F, Lu Y, Zhu Y. 2019. Expression of FSCN1 and FOXM1 are associated with poor prognosis of adrenocortical carcinoma patients. BMC Cancer19(1):1165. DOI 10.1186/s12885-0196389-3.

Liao GB, Li XZ, Zeng S, Liu C, Yang SM, Yang L, Hu CJ, Bai JY. 2018. Regulation of the master regulator FOXM1 in cancer. Cell Communication and Signaling 16(1):57. DOI 10.1186/s12964-018-0266-6. 
Liu K, Luo J, Shao C, Ren Z, Sun S, Zhu Y, Zhou H, Jiang Z, Li X, Gu W, Xu Y, Qiang Y, Ren B, Xu L, Wu H, Shen Y. 2020. Synaptotagmin 12 (SYT12) Gene Expression Promotes Cell Proliferation and Progression of Lung Adenocarcinoma and Involves the Phosphoinositide 3-Kinase (PI3K)/AKT/Mammalian Target of Rapamycin (mTOR) Pathway. Medical Science Monitor 26:e920351. DOI 10.12659/MSM.920351.

Liu L, Wu J, Guo Y, Xie W, Chen B, Zhang Y, Li S, Hua Y, Peng B, Shen S. 2018. Overexpression of FoxM1 predicts poor prognosis of intrahepatic cholangiocarcinoma. Aging (Albany NY)10(12):4120-4140. DOI 10.18632/aging.101706.

Liu X, Liu X, Li J, Ren F. 2019. Identification and Integrated Analysis of Key Biomarkers for Diagnosis and Prognosis of Non-Small Cell Lung Cancer. Medical Science Monitor 25:9280-9289. DOI 10.12659/MSM.918620.

Long T, Liu Z, Zhou X, Yu S, Tian H, Bao Y. 2019. Identification of differentially expressed genes and enriched pathways in lung cancer using bioinformatics analysis. Molecular Medicine Reports 19(3):2029-2040. DOI 10.3892/mmr.2019.9878.

Lu D, Bai X, Zou Q, Gan Z, Lv Y. 2020. Identification of the association between HMMR expression and progression of hepatocellular carcinoma via construction of a co-expression network. Oncology Letters 20(3):2645-2654. DOI 10.3892/ol.2020.11844.

Luo J, Liu K, Yao Y, Sun Q, Zheng X, Zhu B, Zhang Q, Xu L, Shen Y, Ren B. 2019. DMBX1 promotes tumor proliferation and regulates cell cycle progression via repressing OTX2-mediated transcription of p21 in lung adenocarcinoma cell. Cancer Letters 453:45-56. DOI 10.1016/j.canlet.2019.03.045.

Luo J, Yao Y, Ji S, Sun Q, Xu Y, Liu K, Diao Q, Qiang Y, Shen Y. 2019. PITX2 enhances progression of lung adenocarcinoma by transcriptionally regulating WNT3A and activating Wnt/ $\beta$-catenin signaling pathway. Cancer Cell International19:96. DOI 10.1186/s12935019-0800-7.

Luo X, Xu S, Zhong Y, Tu T, Xu Y, Li X, Wang B, Yang F. 2019. High gene expression levels of VEGFA and CXCL8 in the peritumoral brain zone are associated with the recurrence of glioblastoma: A bioinformatics analysis. Oncology Letters8(6):6171-6179. DOI 10.3892/ol.2019.10988.

Mao Y, Xue P, Li L, Xu P, Cai Y, Chu X, Jiang P, Zhu S. 2019. Bioinformatics analysis of mRNA and miRNA microarray to identify the key miRNA-gene pairs in small-cell lung cancer. Molecular Medicine Reports 20(3):2199-2208. DOI 10.3892/mmr.2019.10441.

Qu Y, Cheng B, Shao N, Jia Y, Song Q, Tan B, Wang J. 2020. Prognostic value of immunerelated genes in the tumor microenvironment of lung adenocarcinoma and lung squamous cell carcinoma. Aging (Albany NY)12(6):4757-4777. DOI 10.18632/aging.102871.

Ring A, Nguyen C, Smbatyan G, Tripathy D, Yu M, Press M, Kahn M, Lang JE. 2018. CBP/ $\beta$-Catenin/FOXM1 Is a Novel Therapeutic Target in Triple Negative Breast Cancer. Cancers (Basel)10(12):525. DOI 10.3390/cancers10120525.

Rizzardi AE, Rosener NK, Koopmeiners JS, Isaksson Vogel R, Metzger GJ, Forster CL, Marston LO, Tiffany JR, McCarthy JB, Turley EA, Warlick CA, Henriksen JC, Schmechel SC. 2014.Evaluation of protein biomarkers of prostate cancer aggressiveness. 
487

488

489

490

491

492

493

494

495

496

497

498

499

500

501

502

503

504

505

506

507

508

509

510

511

512

513

514

515

516

517

518

519

520

521

522

523

524

525

526

527

BMC Cancer14:244. DOI 10.1186/1471-2407-14-244.

Senft D, Qi J, Ronai ZA. 2018.Ubiquitin ligases in oncogenic transformation and cancer therapy. Nature Reviews Cancer18(2):69-88. DOI 10.1038/nrc.2017.105.

Shan L, Zhao M, Lu Y, Ning H, Yang S, Song Y, Chai W, Shi X. 2019. CENPE promotes lung adenocarcinoma proliferation and is directly regulated by FOXM1. International Journal of Oncology55(1):257-266. DOI 10.3892/ijo.2019.4805.

Sun Y, Zhang Y, Ren S, Li X, Yang P, Zhu J, Lin L, Wang Z, Jia Y. 2020. Low expression of RGL4 is associated with a poor prognosis and immune infiltration in lung adenocarcinoma patients. International Immunopharmacology83:106454. DOI 10.1016/j.intimp.2020.106454.

Telmer PG, Tolg C, McCarthy JB, Turley EA. 2011. How does a protein with dual mitotic spindle and extracellular matrix receptor functions affect tumor susceptibility and progression? Communicative\&Integrative Biology4(2):182-5. DOI 10.4161/cib.4.2.14270.

Wang H, Ye L, Xing Z, Li H, Lv T, Liu H, Zhang F, Song Y. 2019. CDCA7 promotes lung adenocarcinoma proliferation via regulating the cell cycle. Pathology Researchand Practice215(11):152559. DOI 10.1016/j.prp.2019.152559.

Wang G, Wang H, Zhang C, Liu T, Li Q, Lin X, Xie J, Liu H. 2016. Rac3 regulates cell proliferation through cell cycle pathway and predicts prognosis in lung adenocarcinoma. Tumor Biology37(9):12597-12607. DOI 10.1007/s13277-016-5126-7.

Wang Y, Deng H, Xin S, Zhang K, Shi R, Bao X. 2019. Prognostic and Predictive Value of Three DNA Methylation Signatures in Lung Adenocarcinoma. Frontiers in Genetics 10:349. DOI 10.3389/fgene.2019.00349.

Wang Y, Yun Y, Wu B, Wen L, Wen M, Yang H, Zhao L, Liu W, Huang S, Wen N, Li Y. 2016. FOXM1 promotes reprogramming of glucose metabolism in epithelial ovarian cancer cells via activation of GLUT1 and HK2 transcription. Oncotarget7(30):47985-47997. DOI 10.18632/oncotarget.10103.

Ye S, Liu Y, Fuller AM, Katti R, Ciotti GE, Chor S, Alam MZ, Devalaraja S, Lorent K, Weber K, Haldar M, Pack MA, Eisinger-Mathason TSK. 2020. TGF $\beta$ and Hippo Pathways Cooperate to Enhance Sarcomagenesis and Metastasis through the HyaluronanMediated Motility Receptor (HMMR). Molecular Cancer Research 18(4):560-573. DOI 10.1158/1541-7786.MCR-19-0877.

Yeh MH, Tzeng YJ, Fu TY, You JJ, Chang HT, Ger LP, Tsai KW. 2018. Extracellular Matrix-receptor Interaction Signaling Genes Associated with Inferior Breast Cancer Survival. Anticancer Research38(8):4593-4605. DOI 10.21873/anticanres.

Zhang D, Liu S, Liu Z, Ma C, Jiang Y, Sun C, Li K, Cao G, Lin Z, Wang P, Zhang J, Xu D, Kong F, Zhao S. 2019. Polyphyllin I induces cell cycle arrest in prostate cancer cells via the upregulation of IL6 and P21 expression. Medicine (Baltimore) 98(44):e17743. DOI 10.1097/MD.0000000000017743.

Zhang H, Ren L, Ding Y, Li F, Chen X, Ouyang Y, Zhang Y, Zhang D. 2019. Hyaluronanmediated motility receptor confers resistance to chemotherapy via TGF $\beta / \mathrm{Smad} 2$-induced epithelial-mesenchymal transition in gastric cancer. FASEB Journal 33(5):6365-6377. DOI 
528

529

530

531

532

533

534

535

536

537

538

539

540

541

542

543

544

545

546

547

548

549

550

551

552

553

554

555

556

557

558

559

560

561

562 Figure 7. Prognostic significance of $H M M R$ in LUAD. (A) Difference analysis for the $H M M R$

10.1096/fj.201802186R.

Zhou D, Sun Y, Jia Y, Liu D, Wang J, Chen X, Zhang Y, Ma X. 2019. Bioinformatics and functional analyses of key genes in smoking-associated lung adenocarcinoma. Oncol Lett 18(4):3613-3622. DOI 10.3892/ol.2019.10733.

\section{Figure captions}

Figure 1. Flow chart of identification of $H M M R$ as a prognostic factor for lung adenocarcinoma (LUAD).

Figure 2. Identification of differentially expressed genes (DEGs). Up-regulated (red-colored spots) and down-regulated (green-colored spots) DEGs in LUAD compared with normal lung tissues were identified from four GEO profiles GSE18842 (A), GSE19188 (B), GSE75037 (C) and GSE101929 (D). A total of 115 DEGs, including 38 up-regulated (E) and 77 down-regulated DEGs (F), were collectively shared by the four GEO expression profiles.

Figure 3. Protein-protein interaction (PPI) network establishment and module analysis. (A) The 115 shared DEGs were applied to construct PPI network and perform module analysis. The 24 pink nodes represent DEGs from the key module (cluster 1) with the top MCODE scores(20.92). Biological process (B) and biological pathway enrichment analyses (C) were performed for the 24 DEGs in key module.

Figure 4. Identification of candidate hub genes. (A) The 30 genes with the highest connectivity degrees in the whole PPI network. (B) The 19 DEGs (candidate hub genes) with the highest connectivity degrees in key module network. Differential expression of 19 candidate hub genes in GSE19804 (C) and GSE31210 (D) profiles.

Figure 5. Identification and validation of the hub gene. (A) Among the top three stronglycorrelated factors, $H M M R$ was uniquely common DEG shared by the GSE31210 profile and the TCGA dataset in correlation analyses between gene expression levels and tumor stage. HMMR was significantly associated with tumor stage in GSE31210 profile (B) and TCGA dataset (C). $H M M R$ showed remarkably high expression in LUAD tissues compared with normal lung tissues in non-paired (D) and paired (E) statistical analyses using TCGA dataset. HMMR presented significantly high protein expression in LUAD samples versus normal lung samples according to CPTAC (F) and HPA (G) platforms. The expression of HMMR was further determined by the qRT-PCR (H) and western blot (I) analyses.

Figure 6. Correlation of $H M M R$ expression with the survival of LUAD patients. $H M M R$ was negatively associated with overall survival (OS) (A-E), progression-free survival (PFS) (F-H) and disease-free survival (DFS)(I, J) in LUAD patients. (K) Meta-analysis suggested that $H M M R$ could serve as a prognostic factor for the OS of LUAD patients. 
563 expression in LUAD patients with different demographic and clinical characteristics, including 564 age, gender, tumor stage, primary tumor, lymph node metastasis status, and distant metastasis 565 status. (B) Kaplan-Meier curves for the OS in LUAD patients with specific clinical 566 characteristics. LUAD patients were divided into high and low HMMR expression subgroups 567 based on the median of HMMR expression levels.

568 Figure 8. Co-expression of $H M M R$ and FOXM1. (A) A strong co-expression relationship 569 between $H M M R$ and FOXM1 within LUAD was identified via Oncomine database. Histogram 570 and scatter plots showing the correlation of HMMR with FOXM1 in GSE31210 profile (B) and 571 TCGA dataset (C).

572 Figure 9. Gene set enrichment analysis (GSEA) for HMMR in LUAD. (A) GSEA for the 573 biological process. (B) GSEA for the HALLMARK. (C) GSEA for the KEGG.

574 Figure S1. Enrichment analyses for cellular component and molecullar function. (A) Cellular 575 component (A) and molecular function enrichment analyses (B) were performed for the 24 576 DEGs in key module.

577 Figure S2. Survival analysis for $H M M R$ in GSE31210 profile. High $H M M R$ expression was 578 significantly indicated worse overall survival in LUAD patients. 
Figure 1

Figure 1. Flow chart of identification of $H M M R$ as a prognostic factor for lung adenocarcinoma (LUAD). 


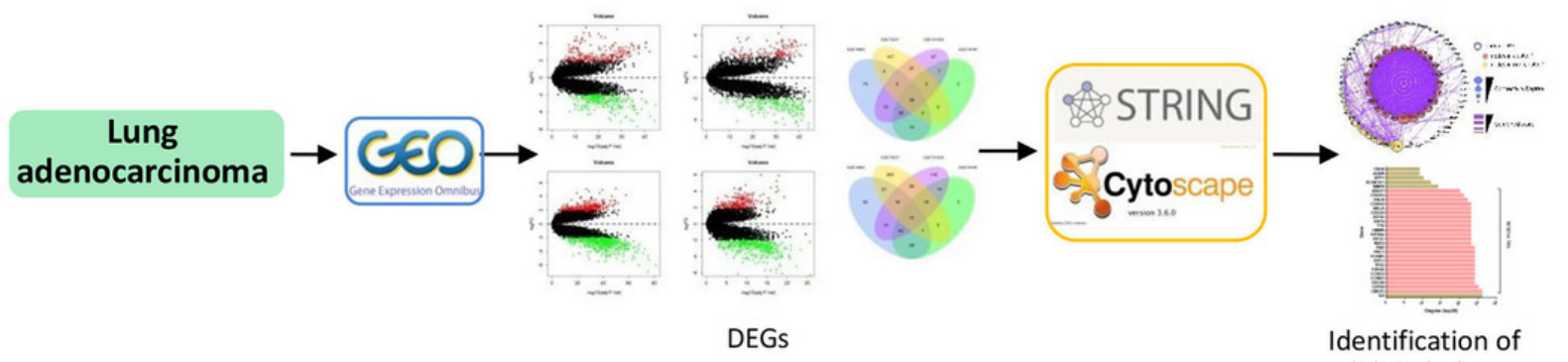
candidate hub genes

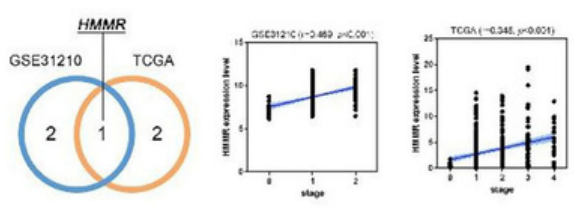

Core gene identification

(HMMR)

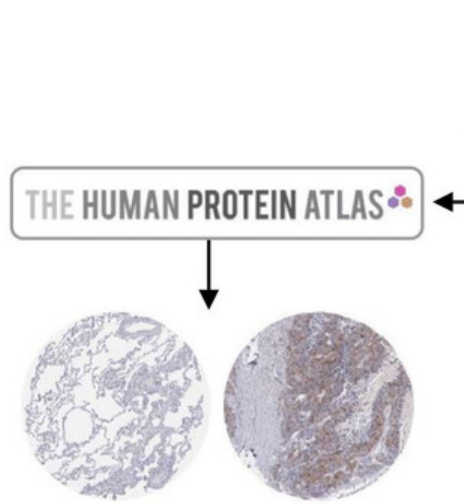

HMMR protein expression

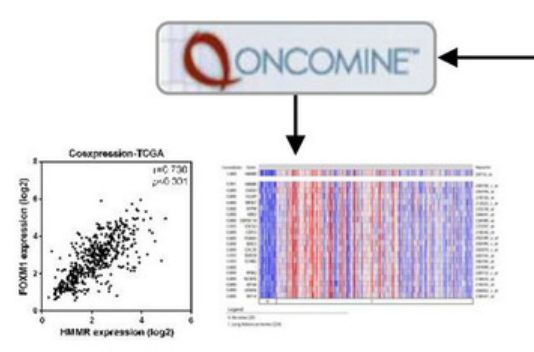

Co-expression analysis

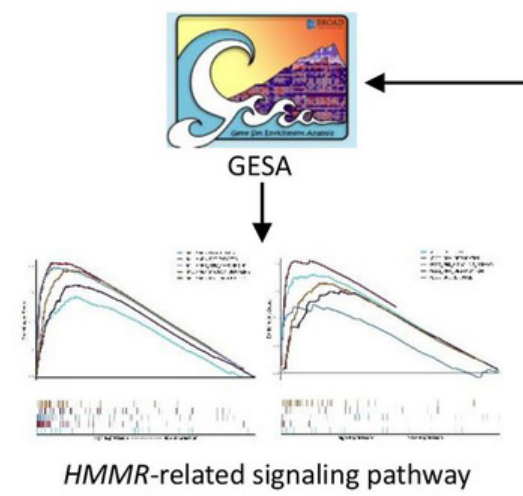

HMMR-related signaling pathway
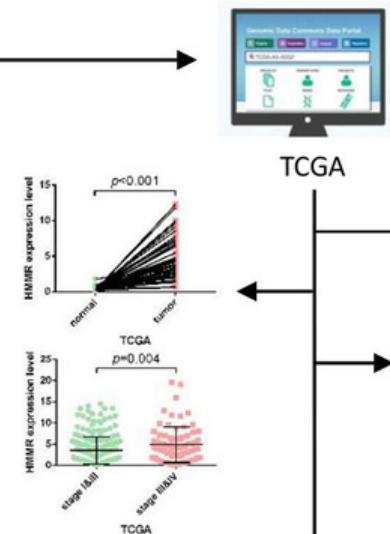

Core gene validation

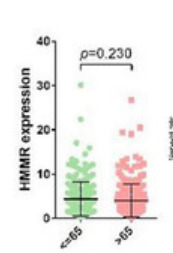

Type

Correlation of HMMR with clinical characteristics

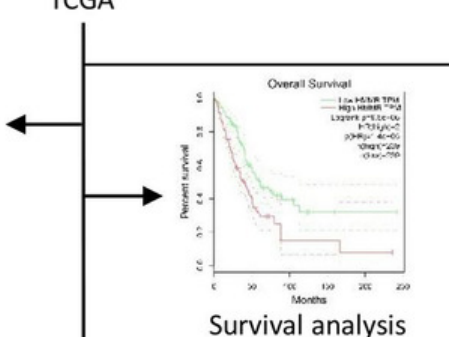

Survival analysis

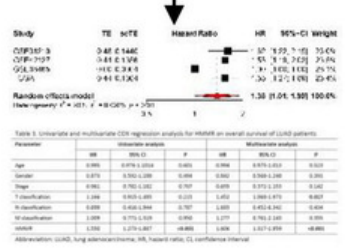

Prognostic analysis

\section{Experiments Validation}
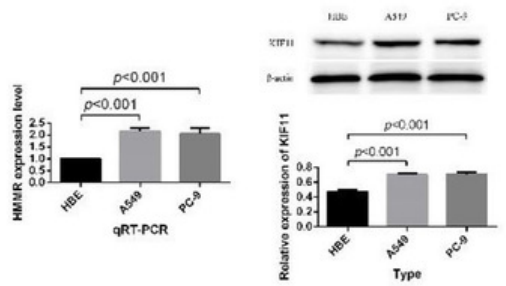

qRT-PCR and western blot analysis 


\section{Figure 2}

Figure 2. Identification of differentially expressed genes (DEGs).

Up-regulated (red-colored spots) and down-regulated (green-colored spots) DEGs in LUAD compared with normal lung tissues were identified from four GEO profiles GSE18842 (A), GSE19188 (B), GSE75037 (C) and GSE101929 (D). The 38 up-regulated (E) and 77 downregulated DEGs (F) were collectively shared by the four GEO expression profiles. 
A

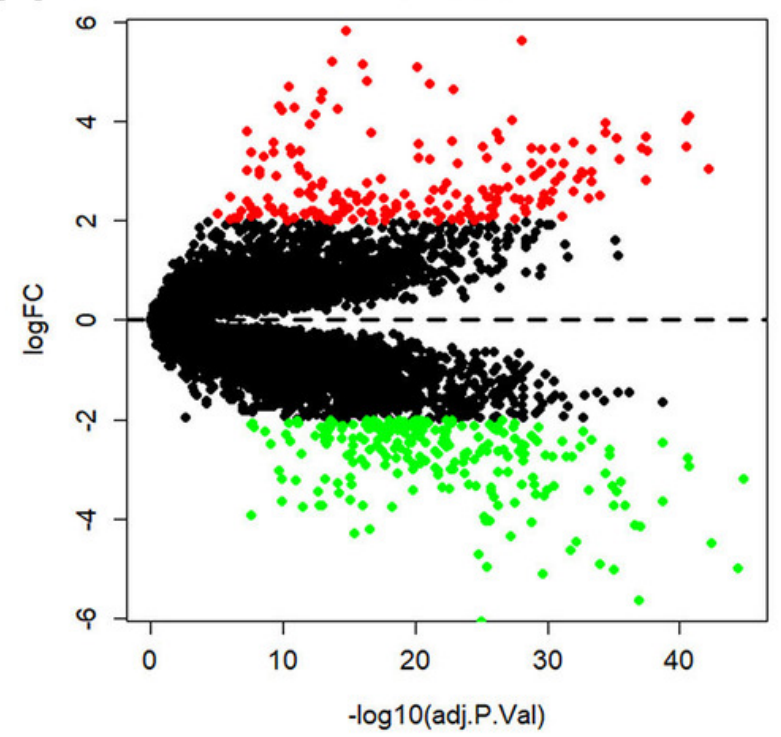

C

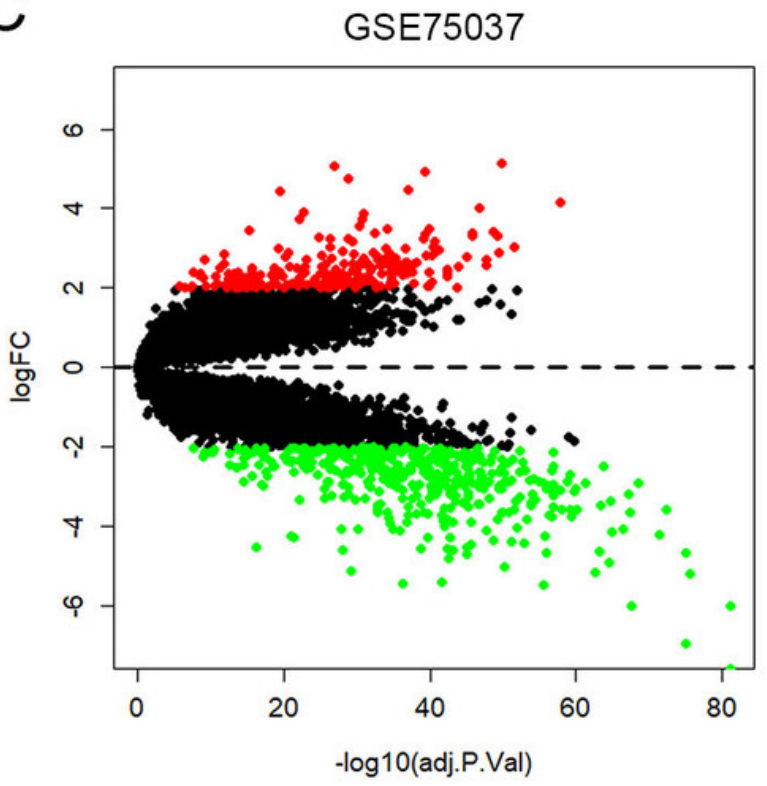

E

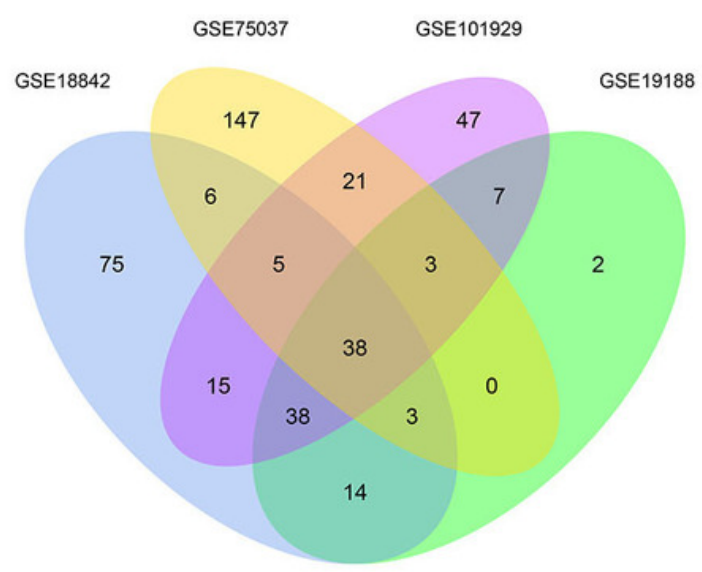

B GSE19188

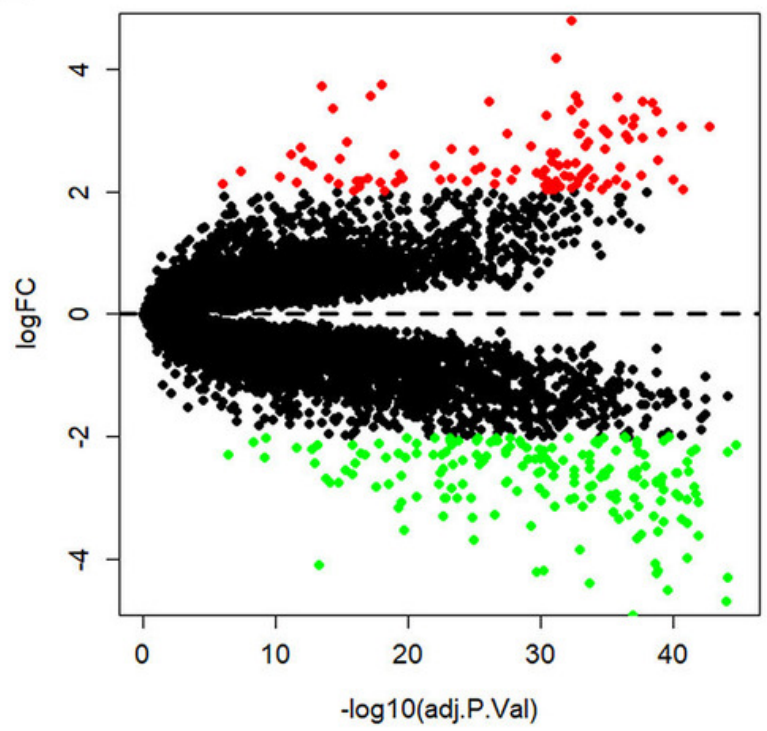

$D$

GSE101929

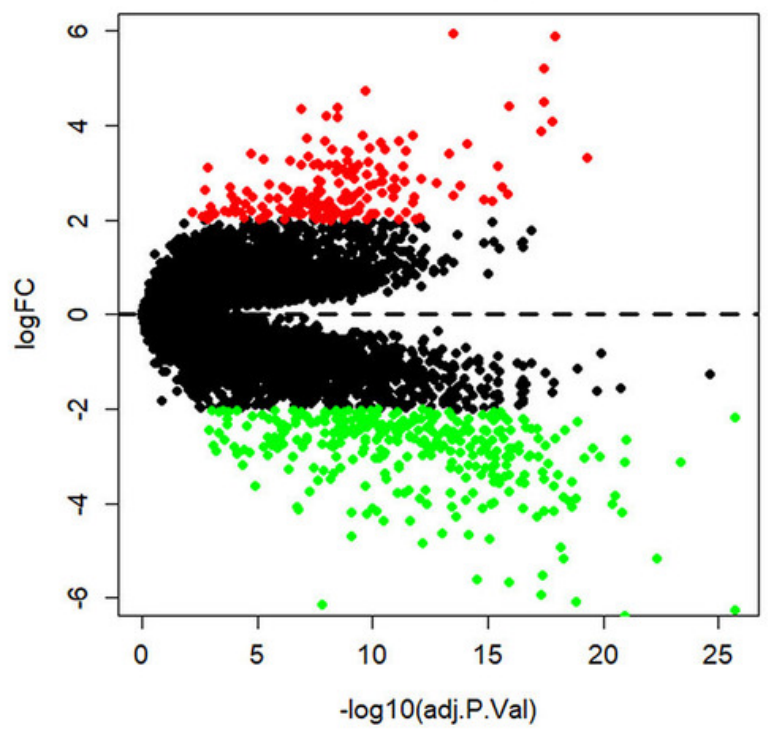

F 


\section{Figure 3}

Figure 3. Construction of protein-protein interaction (PPI) network and module analysis.

(A) The 115 shared DEGs were applied to construct PPI network and perform module analysis. Pink nodes represent the key module (cluster 1 ), MCODE score $=20.92$. Biological process analysis (B) and biological pathway analysis (C) for the corresponding DEGs in the key module. 


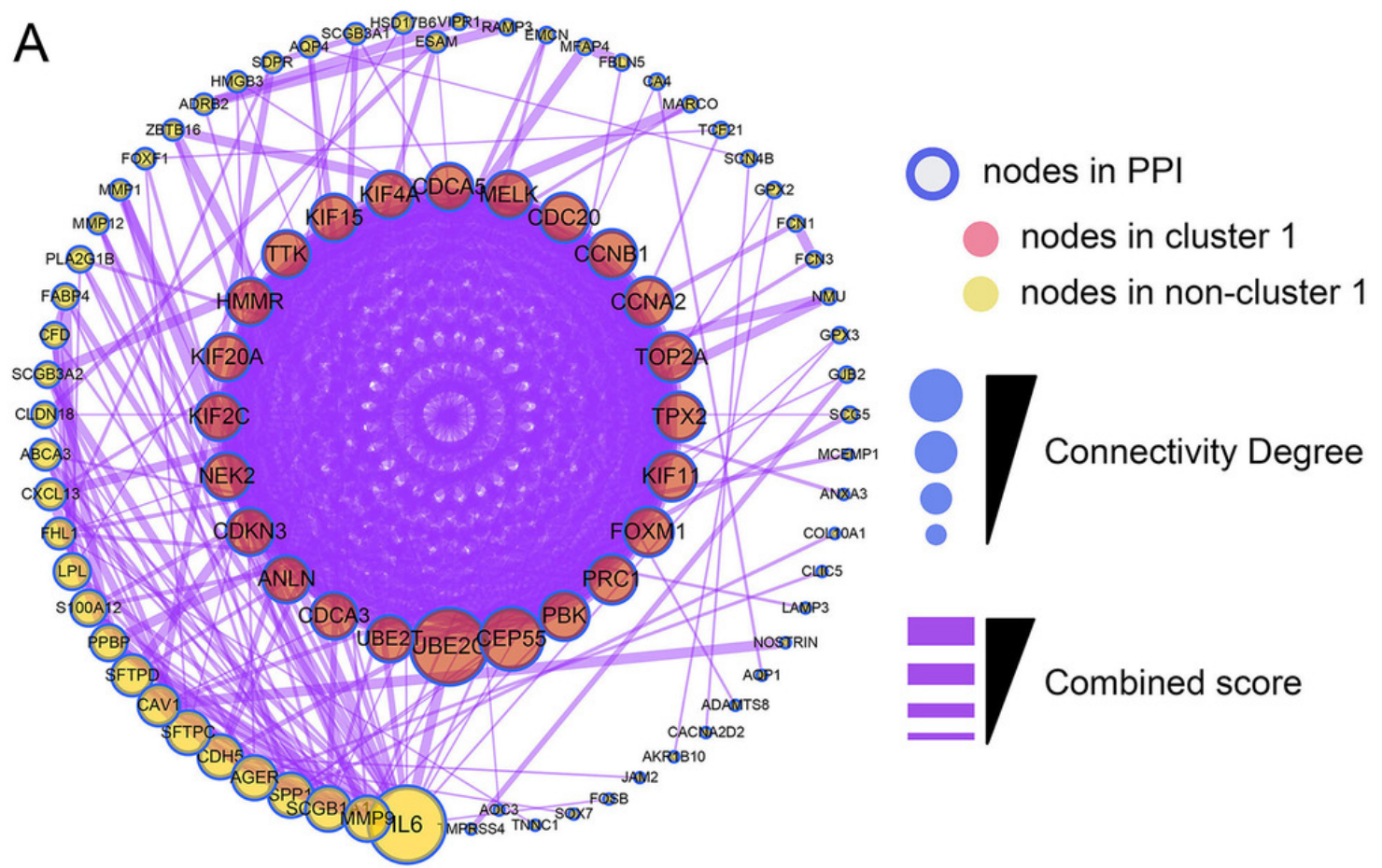

B
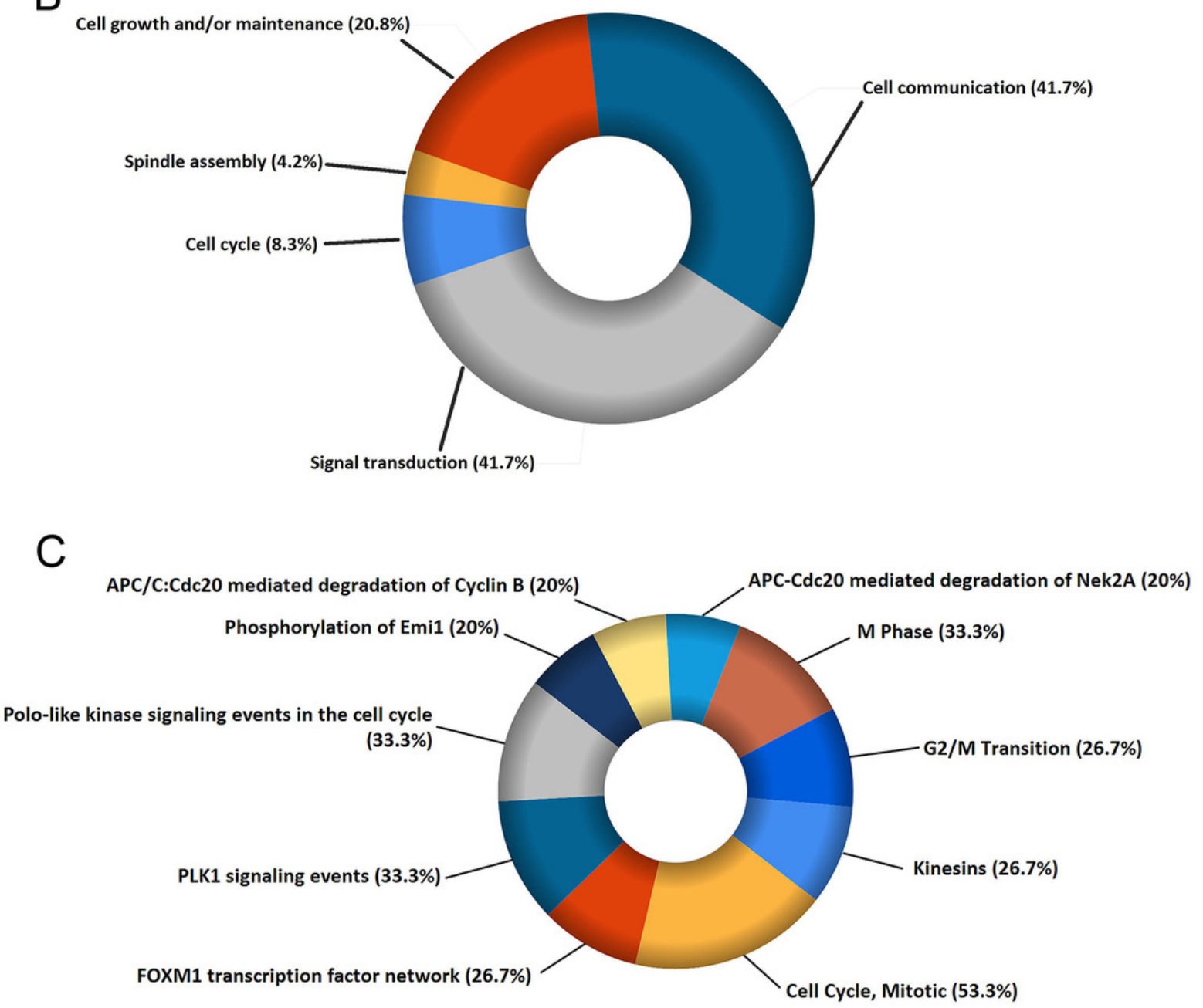
Figure 4

Figure 4. Identification of candidate hub genes.

(A) The 30 genes with the highest connectivity degrees in the PPI network. The 19 DEGs (candidate hub genes) with the highest connectivity degrees in the key module (B) were further validated in the GSE19804 (C) and GSE31210 (D) profiles. 
A

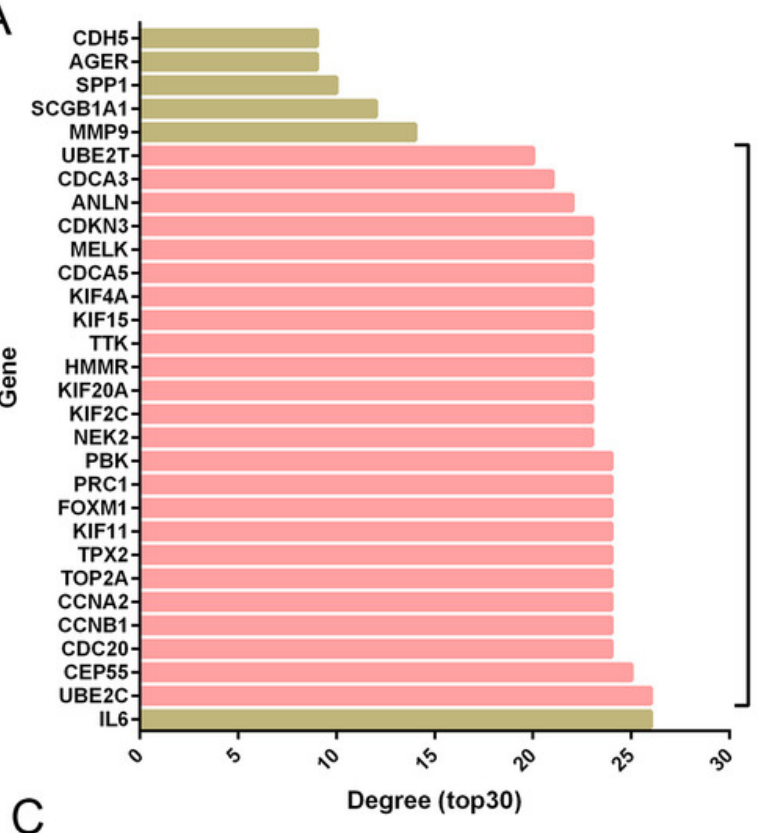

B

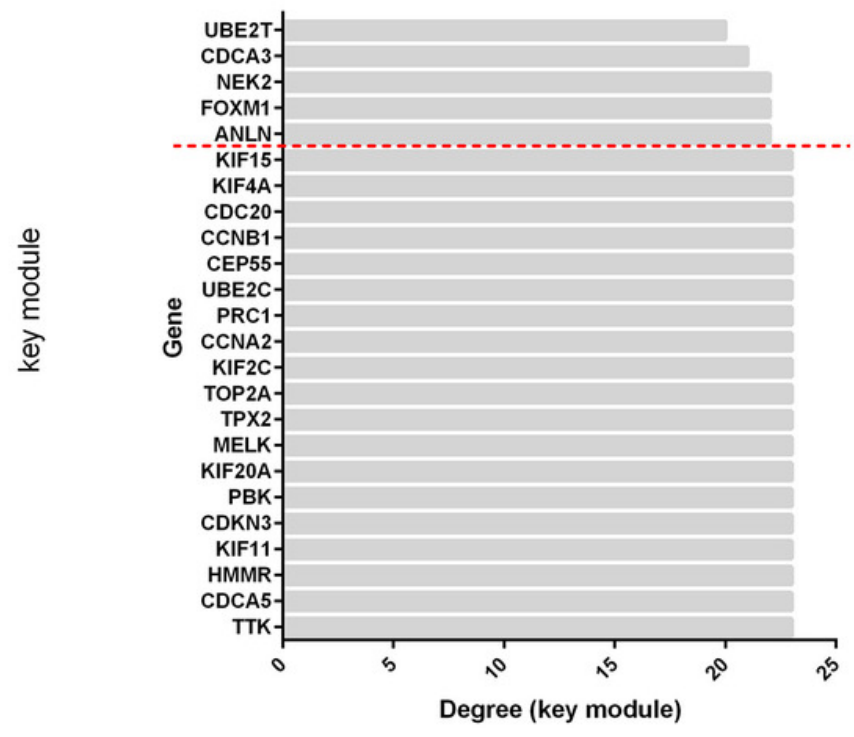

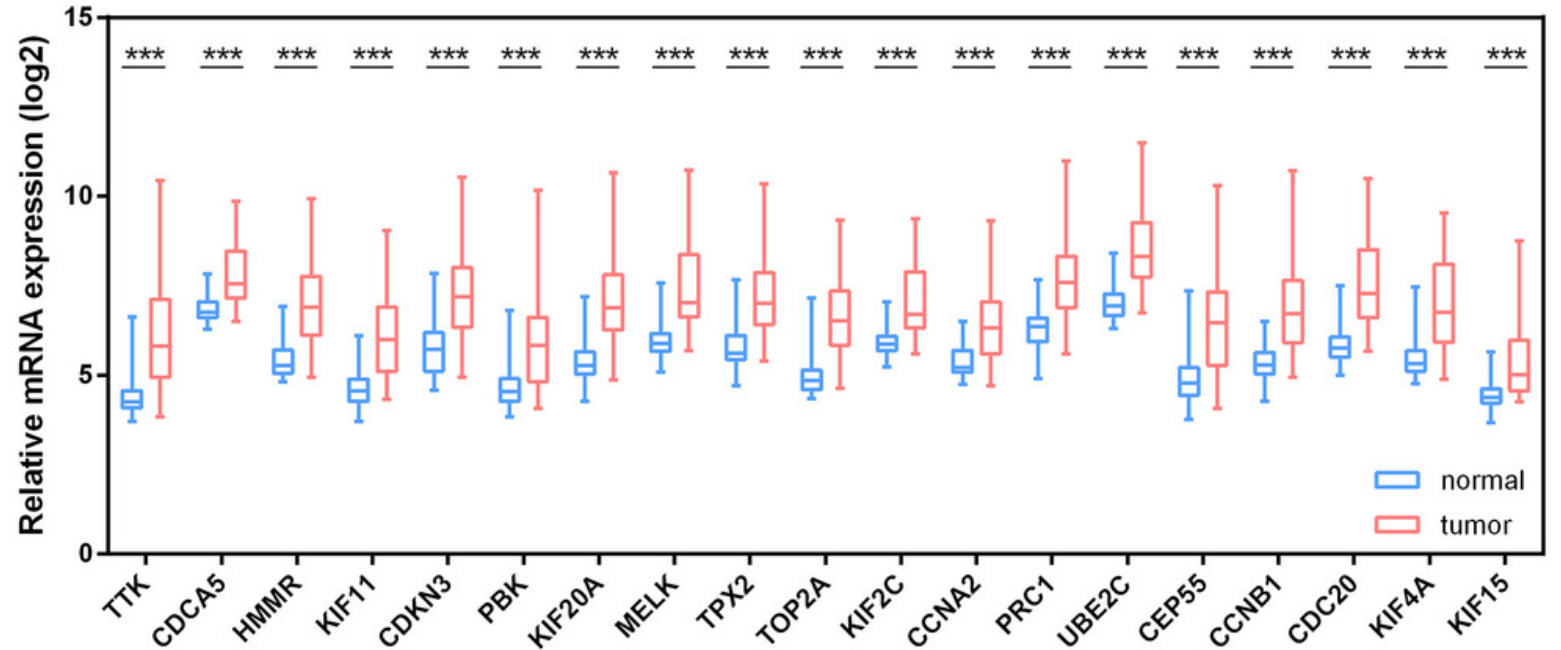

$\mathrm{D}$ Gene (GSE19804)

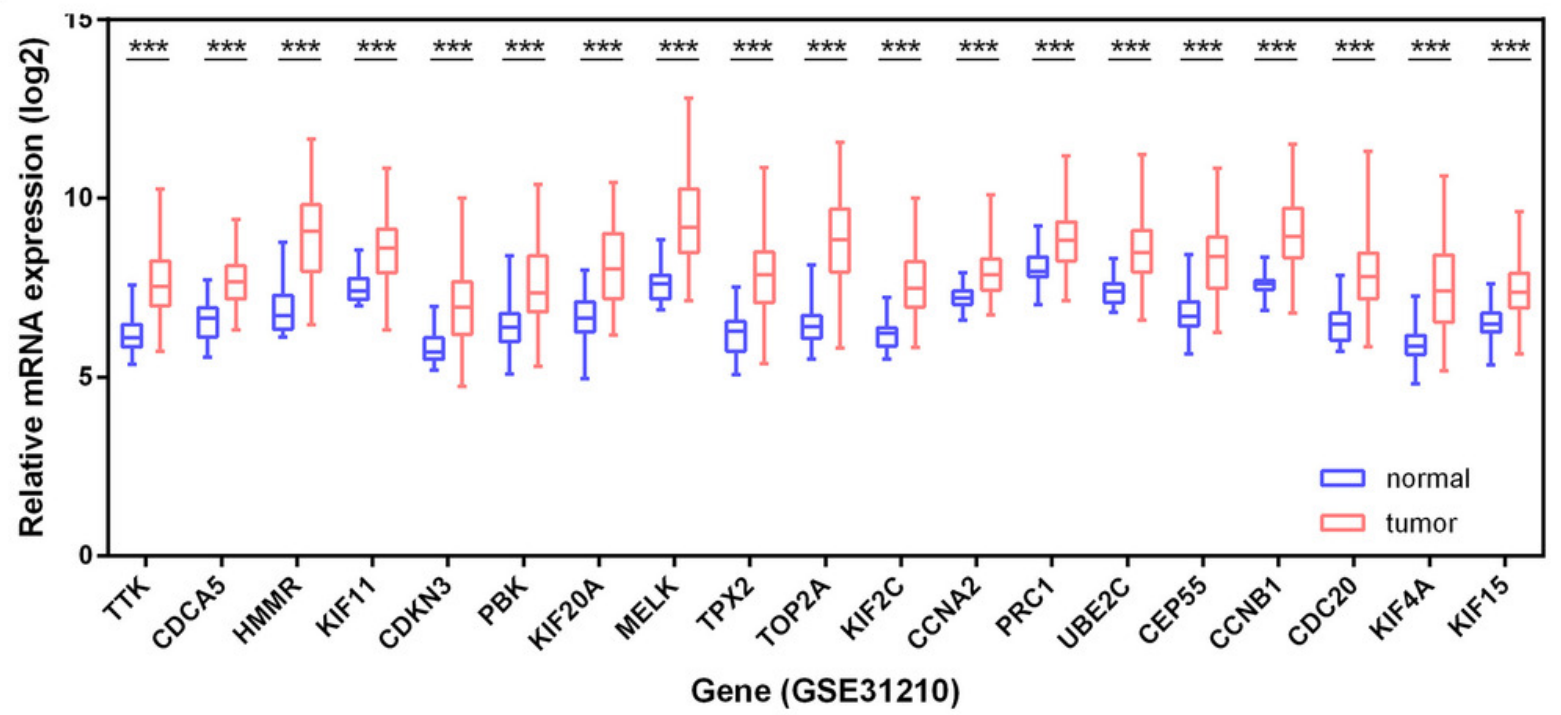




\section{Figure 5}

Figure 5. Identification and validation of the hub gene.

(A) HMMR was uniquely shared by the GSE31210 profile and the TCGA dataset among the top three strongly-correlated factors. (B, C) HMMR was significantly associated with tumor stage. (D-G) HMMR showed remarkably high expression in LUAD tissues compared with normal lung tissues. The expression of HMMR was further determined by the qRT-PCR $(\mathrm{H})$ and western blot (I) analyses. 

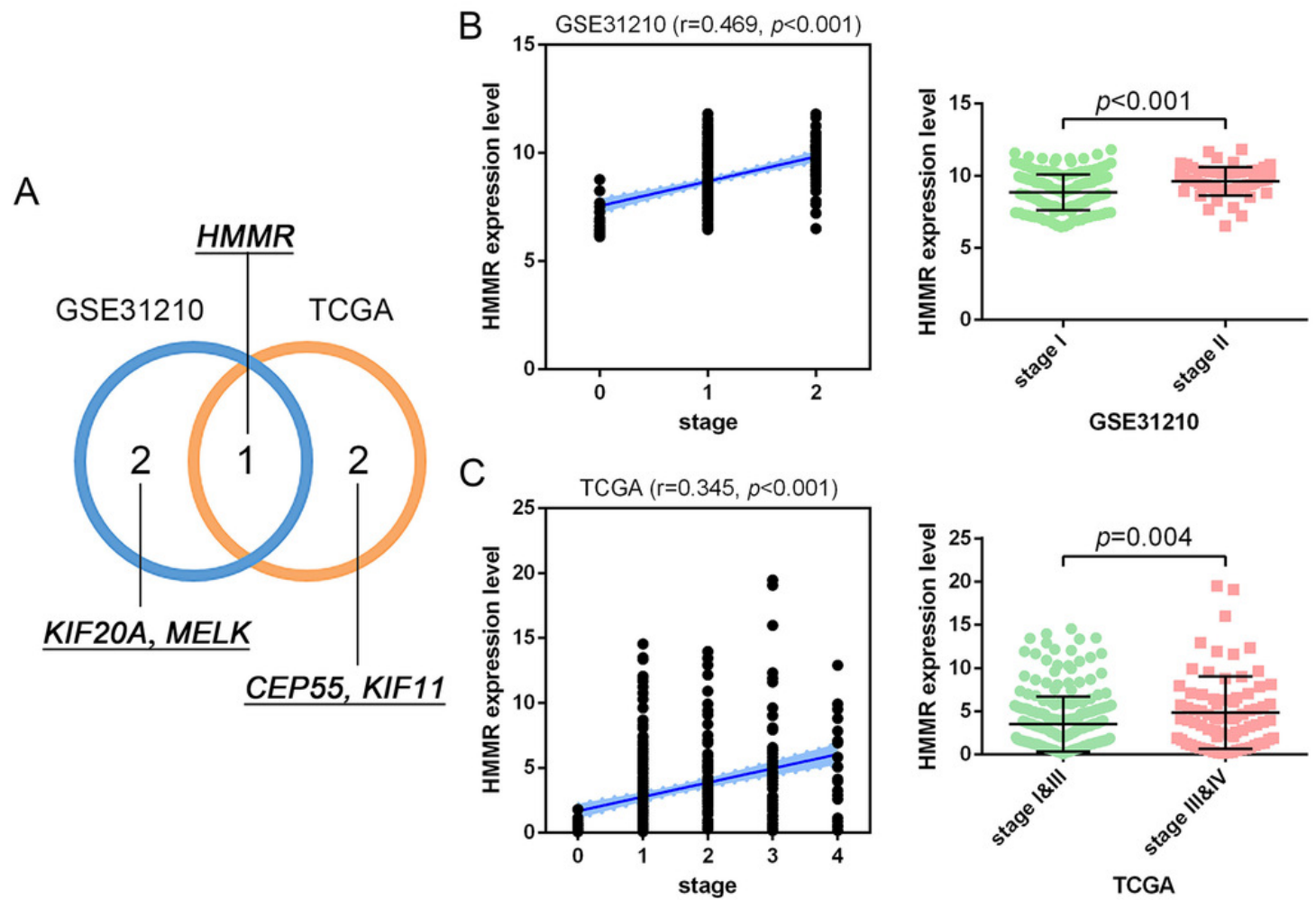

D

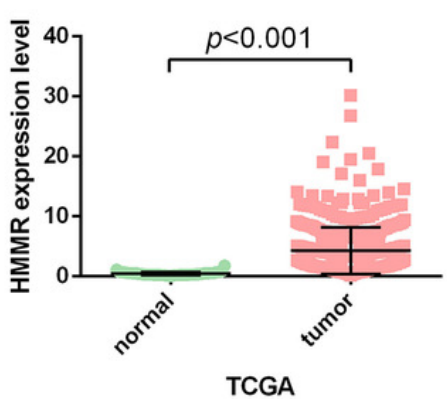

G

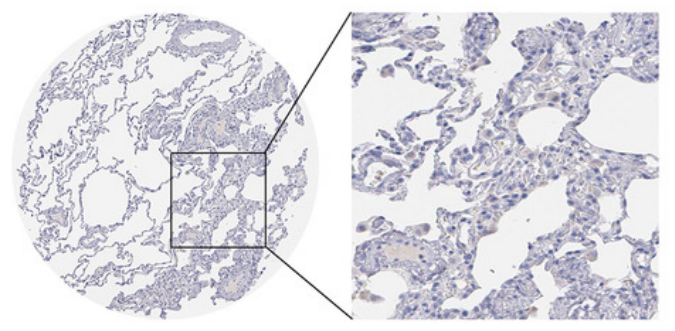

normal lung tissue

(CAB002433, Male, age: 59)

$\mathrm{H}$

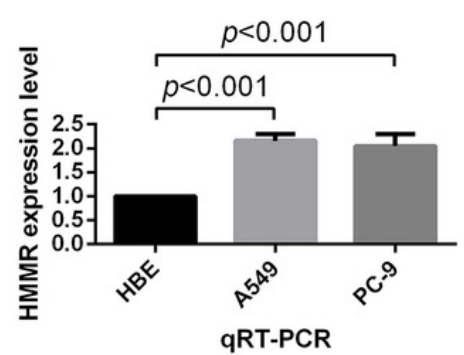

$E$

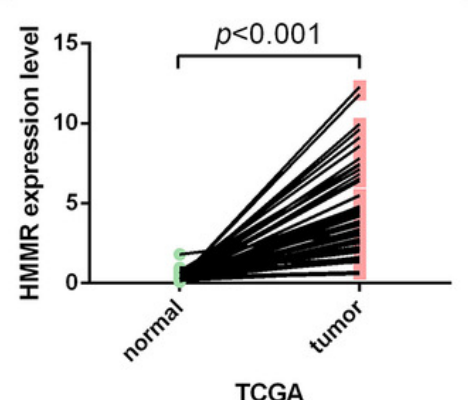

TCGA
F

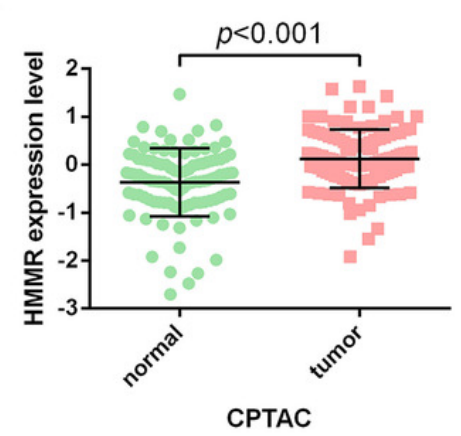

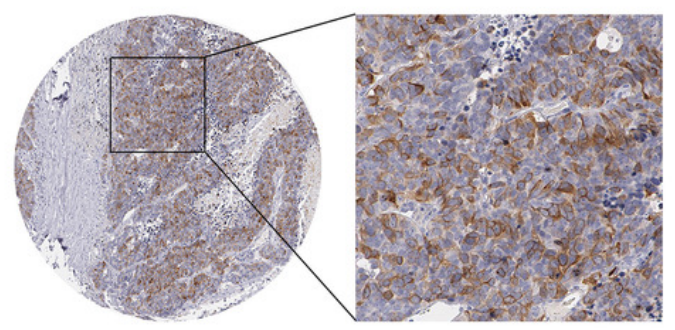

LUAD tissue

(CAB002433, Male, age: 60)
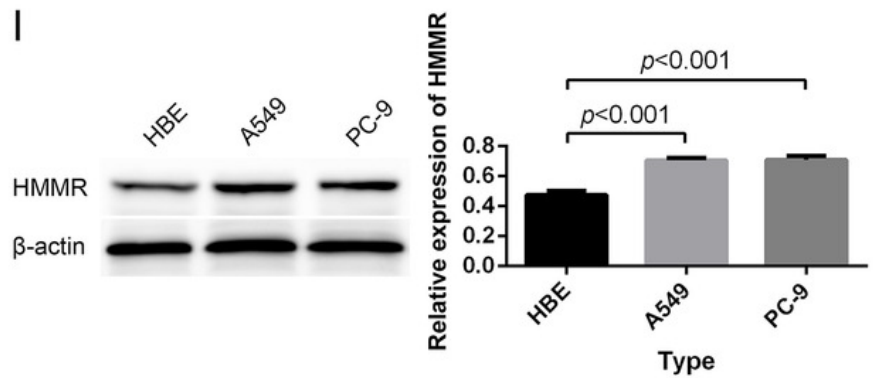

PeerJ reviewing PDF | (2021:04:60512:1:1:NEW 29 Oct 2021) 


\section{Figure 6}

Figure 6. Correlation of HMMR expression with the survival of LUAD patients.

HMMR was negatively associated with overall survival (OS) (A-C), progression-free survival (PFS) (D, E) and disease-free survival (DFS) (F) in LUAD patients. Kaplan-Meier Plotter (G, H) and GEPIA (I, J) datasets showed that high HMMR expression significantly deteriorated OS, PFS, and DFS in LUAD patients. (K) Meta-analysis suggested that HMMR could serve as a prognostic factor for the OS of LUAD patients. 
A

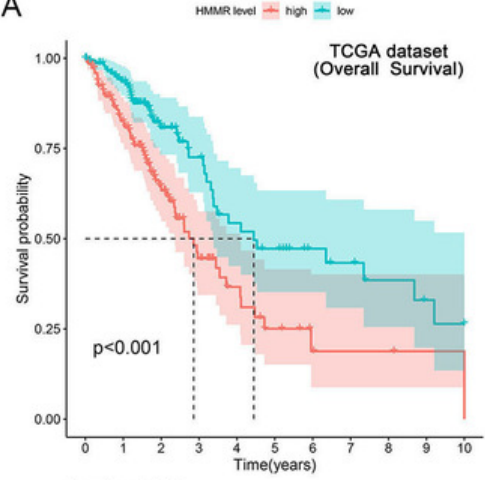

D. Number at risk

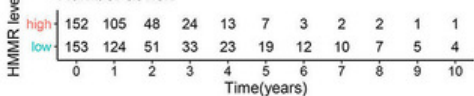

D

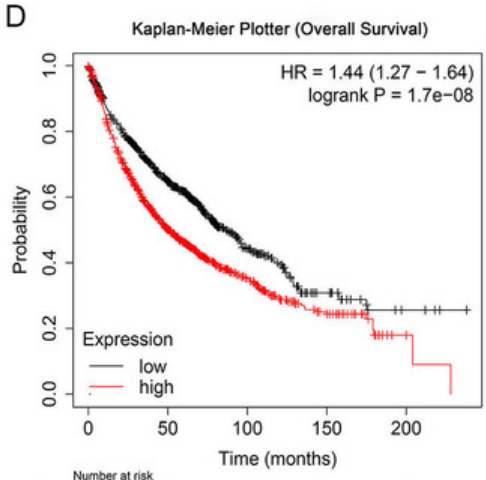

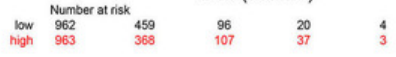

G
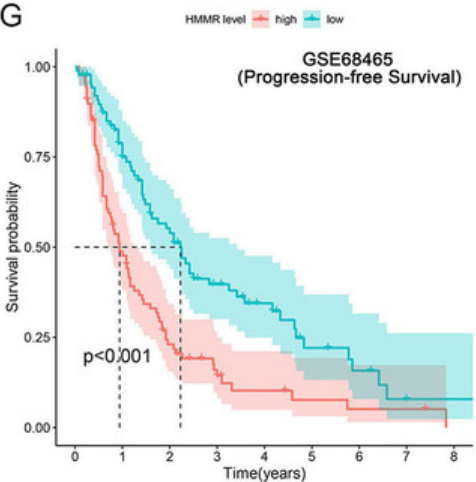

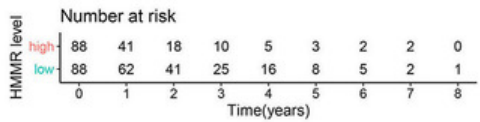

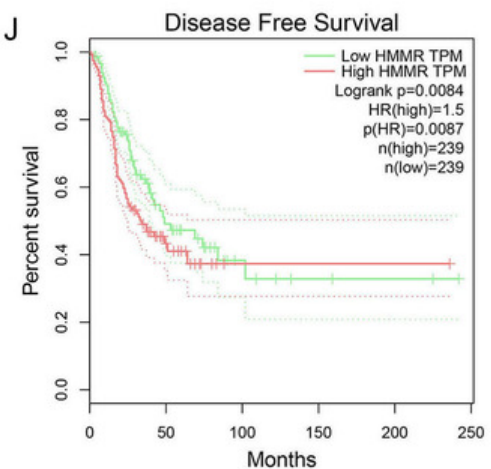

B

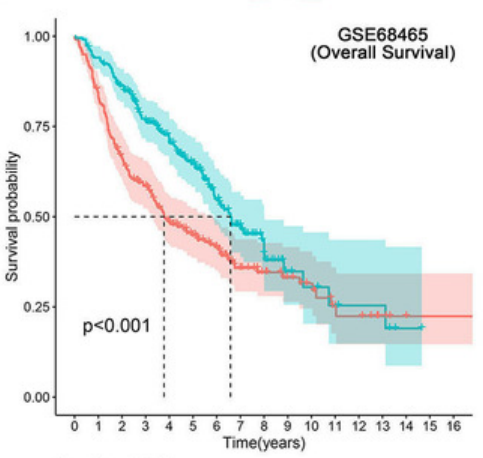

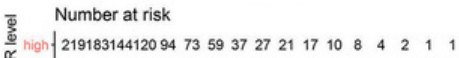

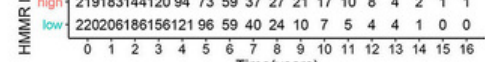

E

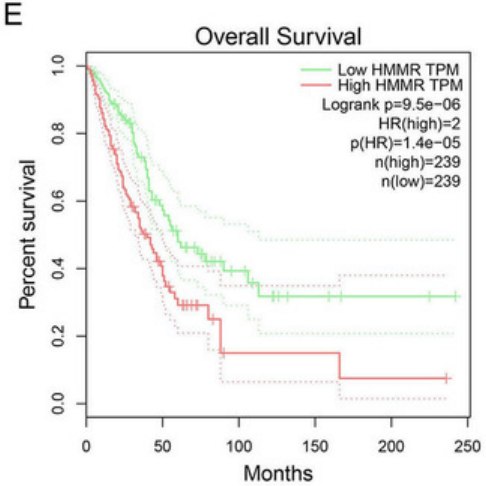

$\mathrm{H}$

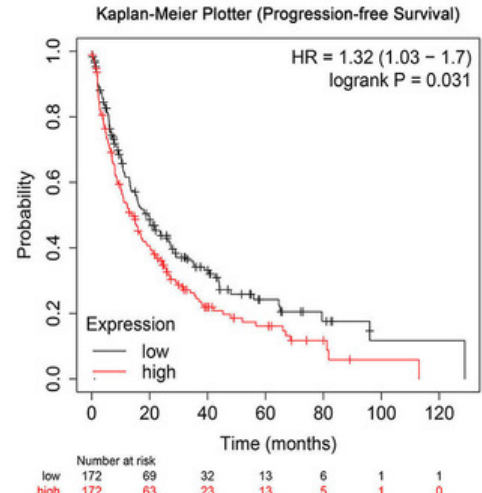

K

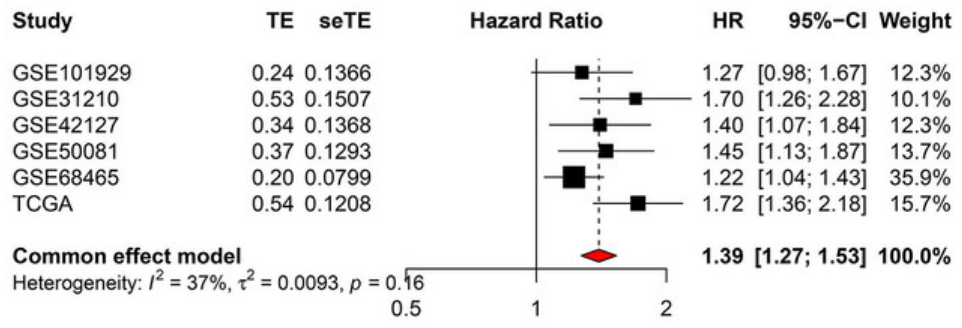

C

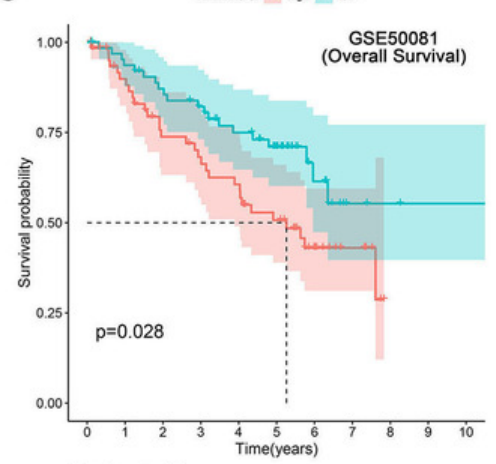

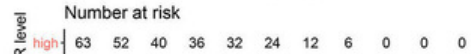

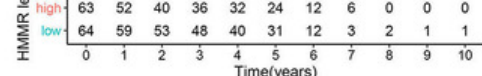

$\mathrm{F}$

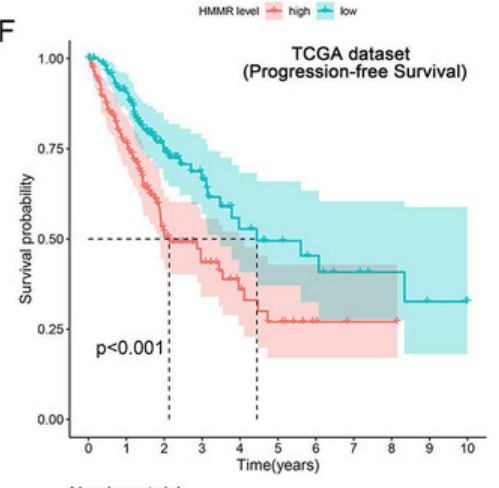

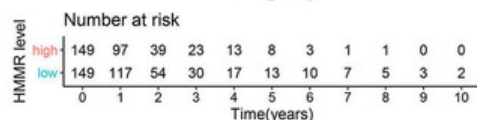

।

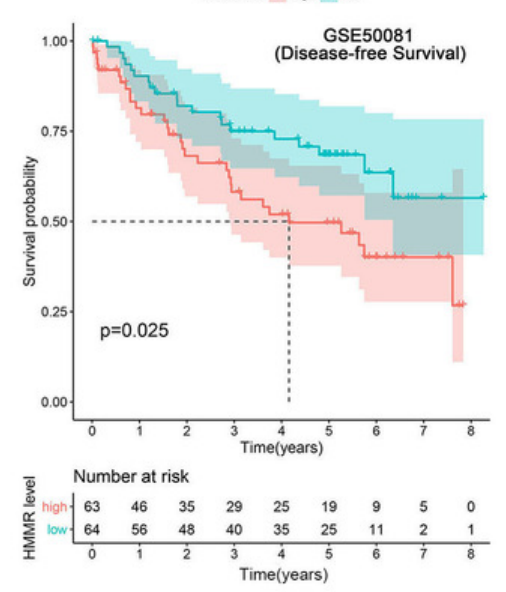




\section{Figure 7}

Figure 7. Prognostic significance of HMMR in LUAD.

(A) Difference analysis for the HMMR expression in LUAD patients with different demographic and clinical characteristics, including age, gender, tumor stage, primary tumor, lymph node metastasis status, and distant metastasis status. (B) Kaplan-Meier curves for the OS in LUAD patients with specific clinical characteristics. LUAD patients were divided into high and low HMMR expression subgroups based on the median of HMMR expression levels. 


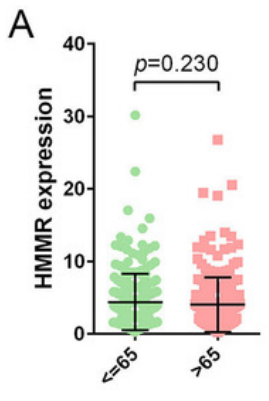

Type

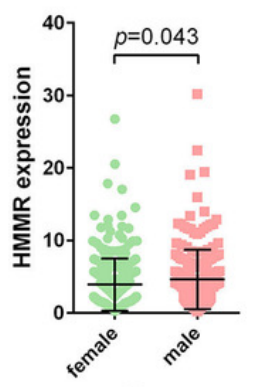

Type
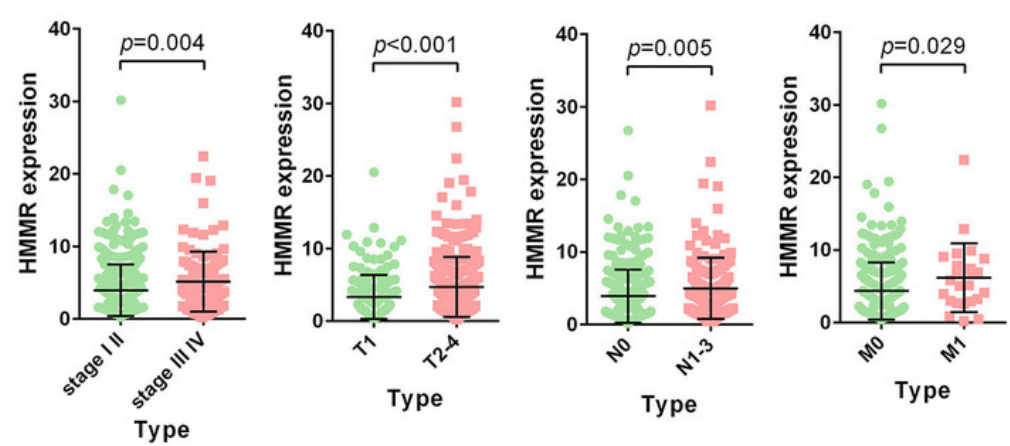

B
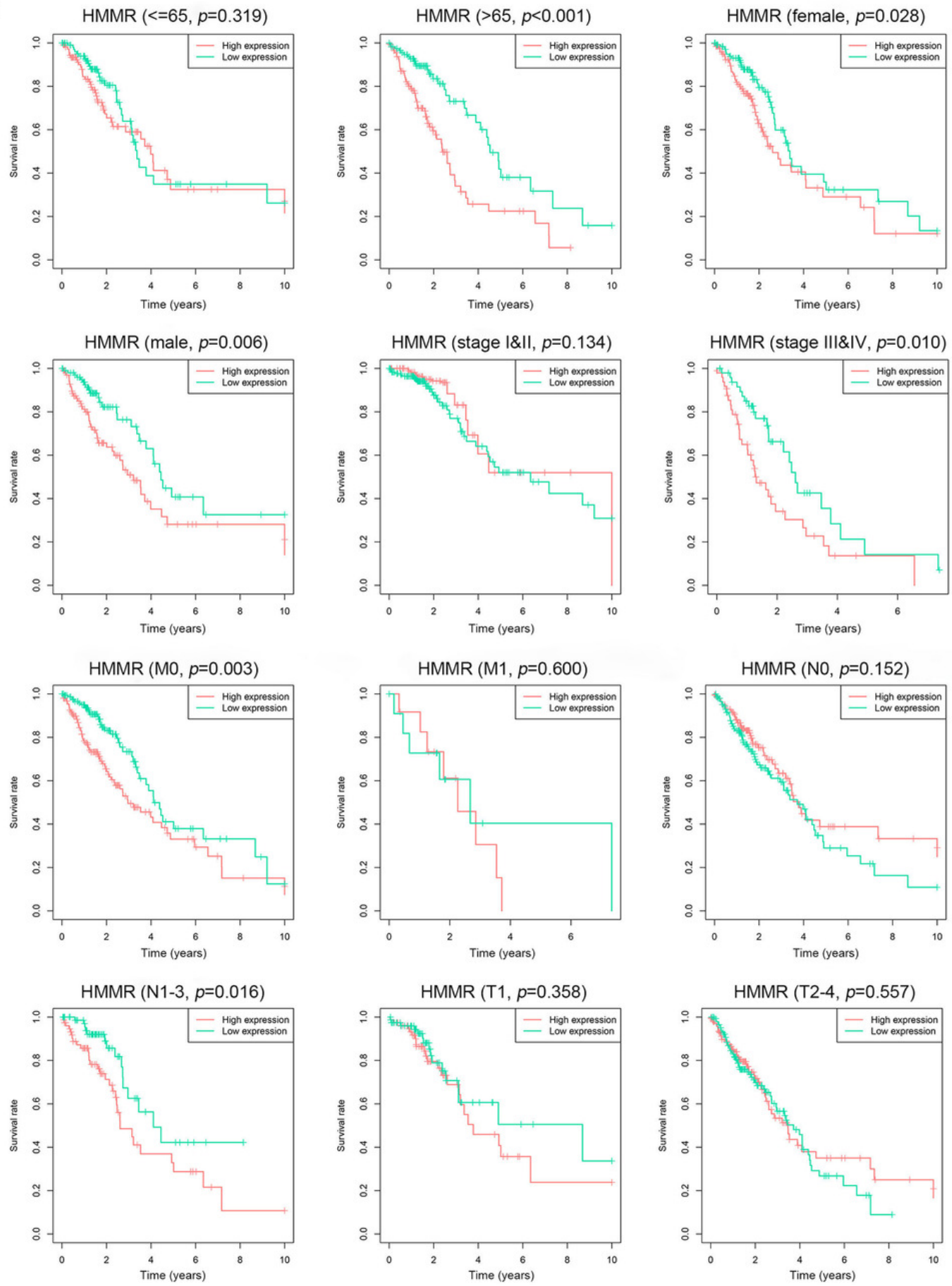
Figure 8

Figure 8. Co-expression of HMMR and FOXM1.

A strong co-expression relationship between HMMR and FOXM1 within LUAD was identified in the Oncomine database (A) and validated in the GSE31210 profile (B) and the TCGA dataset (C).

A

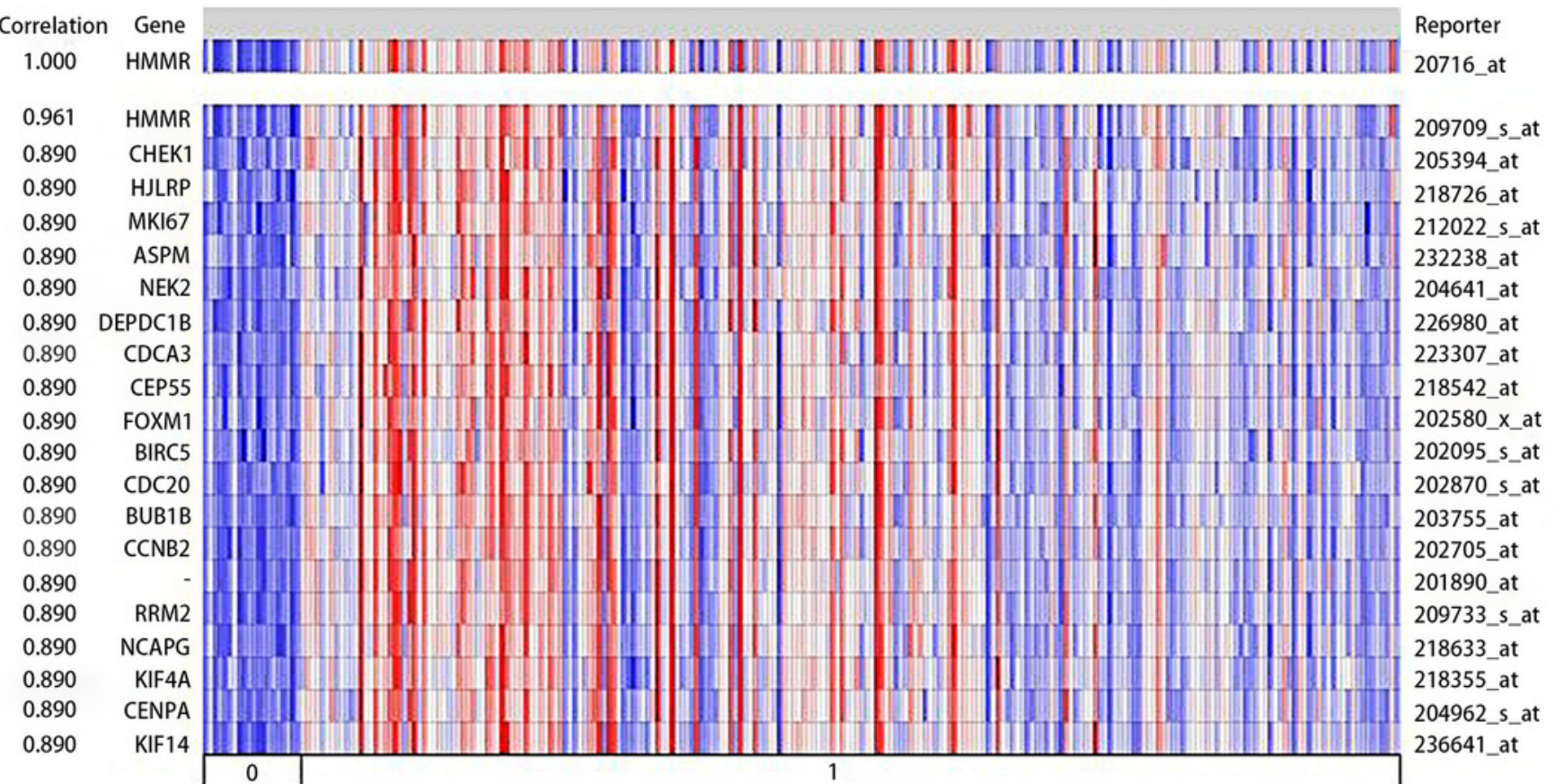

\section{Legend}

0 . No value (20)

1. Lung Adenocarcinoma (226)

B

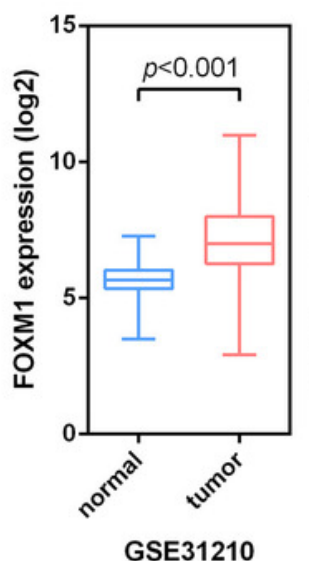

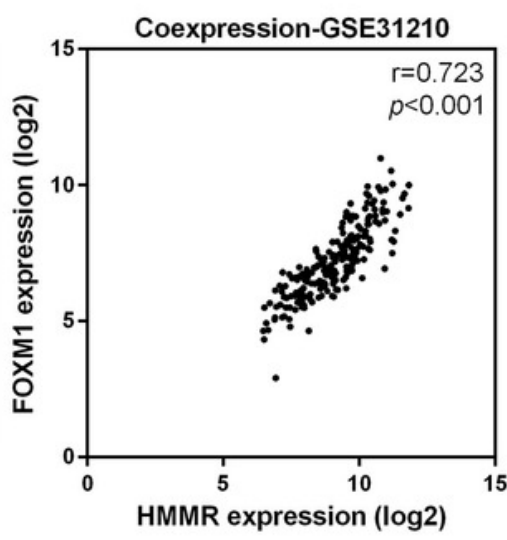

HMMR expression (log2)

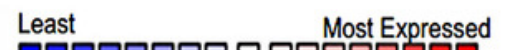

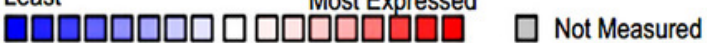

C

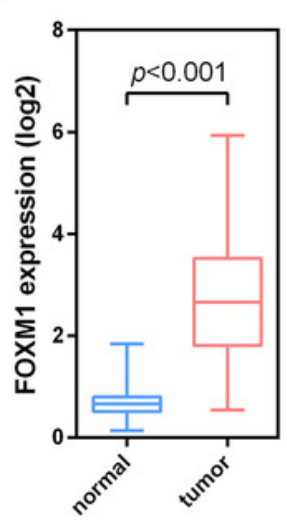

TCGA

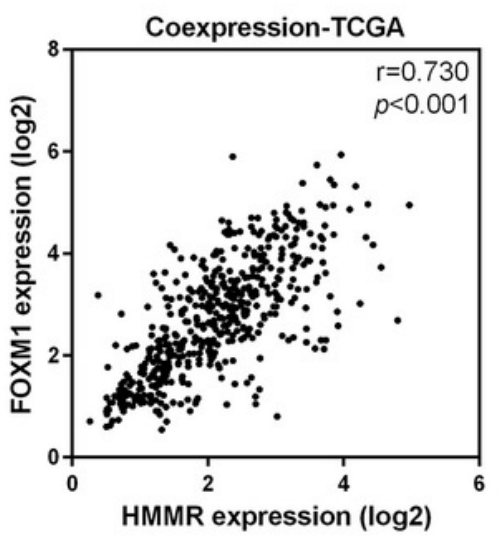

HMMR expression (log2) 
Figure 9

Figure 9. Gene set enrichment analysis (GSEA) for upregulated genes in the high HMMR expression group.

(A) Results of biological process enrichment analysis. (B) Results of HALLMARK enrichment analysis. (C) Results of KEGG enrichment analysis. 

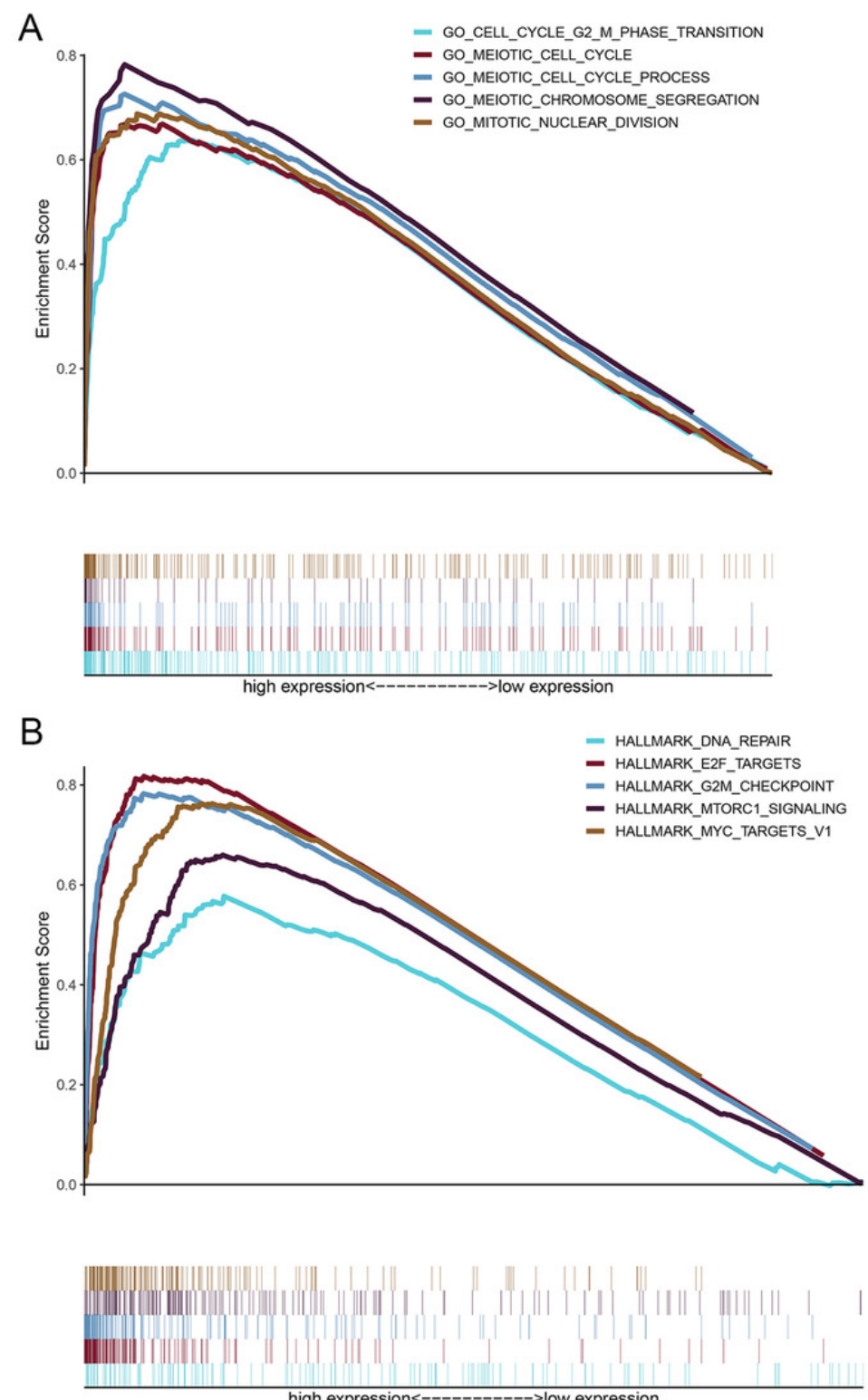

C
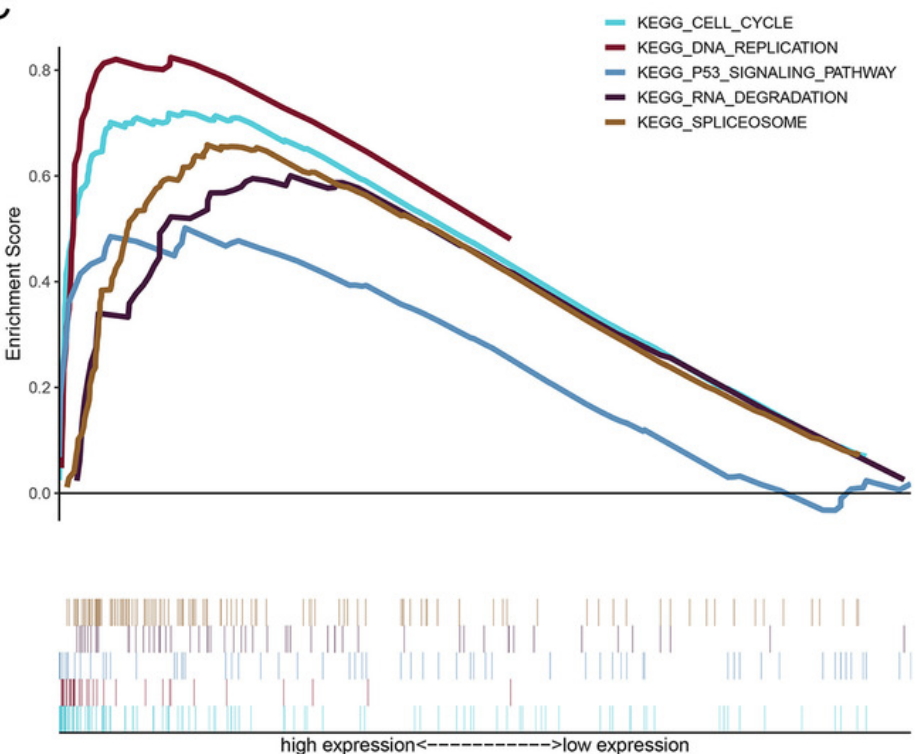

Peer) reviewing PDF | (2021:04:60512:1:1:NEW 29 Oct 2021) 


\section{Table $\mathbf{1}$ (on next page)}

Table 1. Correlation of candidate hub genes with tumor stage. 
Table 1. Correlation of candidate hub genes with tumor stage.

\begin{tabular}{|c|c|c|c|c|c|c|c|}
\hline \multicolumn{4}{|c|}{ GSE31210 profile } & \multicolumn{4}{|c|}{ TCGA dataset } \\
\hline NO. & gene & R (stage) & $\mathbf{P}$ & NO. & gene & R (stage) & $\mathbf{P}$ \\
\hline 1 & $H M M R$ & 0.469 & $<0.001$ & 1 & CEP55 & 0.235 & $<0.001$ \\
\hline 2 & $K I F 20 A$ & 0.449 & $<0.001$ & 2 & KIF11 & 0.232 & $<0.001$ \\
\hline 3 & $M E L K$ & 0.448 & $<0.001$ & 3 & $H M M R$ & 0.228 & $<0.001$ \\
\hline 4 & $C C N B 1$ & 0.447 & $<0.001$ & 4 & PRC1 & 0.198 & $<0.001$ \\
\hline 5 & TOP $2 A$ & 0.445 & $<0.001$ & 5 & $C C N B 1$ & 0.194 & $<0.001$ \\
\hline 6 & $\mathrm{KIF} 2 \mathrm{C}$ & 0.443 & $<0.001$ & 6 & $K I F 4 A$ & 0.193 & $<0.001$ \\
\hline 7 & $T P X 2$ & 0.433 & $<0.001$ & 7 & $C D C A 5$ & 0.185 & $<0.001$ \\
\hline 8 & $C D C 20$ & 0.432 & $<0.001$ & 8 & $P B K$ & 0.176 & $<0.001$ \\
\hline 9 & $K I F 4 A$ & 0.429 & $<0.001$ & 9 & $M E L K$ & 0.170 & $<0.001$ \\
\hline 10 & CEP55 & 0.423 & $<0.001$ & 10 & $K I F 20 A$ & 0.169 & $<0.001$ \\
\hline 11 & $T T K$ & 0.417 & $<0.001$ & 11 & $T T K$ & 0.159 & $<0.001$ \\
\hline 12 & $C D C A 5$ & 0.417 & $<0.001$ & 12 & $C D K N 3$ & 0.151 & $<0.001$ \\
\hline 13 & KIF 11 & 0.409 & $<0.001$ & 13 & TOP $2 A$ & 0.141 & 0.002 \\
\hline 14 & $C D K N 3$ & 0.405 & $<0.001$ & 14 & CCNA2 & 0.137 & 0.003 \\
\hline 15 & $C C N A 2$ & 0.380 & $<0.001$ & 15 & $C D C 20$ & 0.129 & 0.005 \\
\hline 16 & $U B E 2 C$ & 0.376 & $<0.001$ & 16 & $U B E 2 C$ & 0.106 & 0.021 \\
\hline 17 & PRC1 & 0.372 & $<0.001$ & 17 & $T P X 2$ & 0.106 & 0.021 \\
\hline 18 & $P B K$ & 0.365 & $<0.001$ & 18 & $\mathrm{KIF} 2 \mathrm{C}$ & 0.098 & 0.033 \\
\hline 19 & KIF 15 & 0.364 & $<0.001$ & 19 & KIF 15 & 0.098 & 0.033 \\
\hline
\end{tabular}

2 Abbreviation: $\mathrm{R}$ : correlation coefficient; $\mathrm{P}: p$-value. 


\section{Table 2 (on next page)}

Table 2. Cox analysis of HMMR on OS and PFS in LUAD patients 
Table 2. Cox analysis of HMMR on OS and PFS in LUAD patients

\begin{tabular}{|c|c|c|c|c|c|c|}
\hline \multirow{2}{*}{ Parameter } & \multicolumn{3}{|c|}{ univariate Cox analysis } & \multicolumn{3}{|c|}{ multivariate Cox analysis } \\
\hline & HR & $95 \% \mathrm{CI}$ & $\mathbf{P}$ & HR & $95 \%$ CI & $\mathbf{P}$ \\
\hline \multicolumn{7}{|l|}{ TCGA (OS) } \\
\hline$H M M R$ & 1.550 & $1.273 \sim 1.887$ & $<0.001$ & 1.606 & $1.317 \sim 1.959$ & $<0.001$ \\
\hline age & 0.995 & $0.976 \sim 1.014$ & 0.601 & 0.994 & $0.975 \sim 1.013$ & 0.523 \\
\hline gender & 0.873 & $0.592 \sim 1.288$ & 0.494 & 0.842 & $0.568 \sim 1.248$ & 0.391 \\
\hline stage & 0.961 & $0.782 \sim 1.182$ & 0.707 & 0.655 & $0.372 \sim 1.153$ & 0.142 \\
\hline $\mathrm{T}$ classification & 1.166 & $0.915 \sim 1.485$ & 0.215 & 1.452 & $1.068 \sim 1.973$ & 0.017 \\
\hline M classification & 0.899 & $0.416 \sim 1.944$ & 0.787 & 1.693 & $0.452 \sim 6.342$ & 0.434 \\
\hline $\mathrm{N}$ classification & 1.009 & $0.771 \sim 1.319$ & 0.950 & 1.277 & $0.761 \sim 2.143$ & 0.355 \\
\hline \multicolumn{7}{|l|}{ TCGA (PFS) } \\
\hline$H M M R$ & 1.627 & $1.315 \sim 2.014$ & $<0.001$ & 1.693 & $1.368 \sim 2.094$ & $<0.001$ \\
\hline age & 0.992 & $0.973 \sim 1.012$ & 0.429 & 0.993 & $0.973 \sim 1.013$ & 0.491 \\
\hline gender & 0.825 & $0.557 \sim 1.221$ & 0.336 & 0.800 & $0.536 \sim 1.194$ & 0.274 \\
\hline stage & 0.870 & $0.703 \sim 1.075$ & 0.197 & 0.641 & $0.359 \sim 1.146$ & 0.134 \\
\hline $\mathrm{T}$ classification & 1.075 & $0.832 \sim 1.389$ & 0.581 & 1.371 & $0.978 \sim 1.921$ & 0.067 \\
\hline M classification & 0.610 & $0.247 \sim 1.508$ & 0.285 & 1.556 & $0.347 \sim 6.972$ & 0.564 \\
\hline $\mathrm{N}$ classification & 0.910 & $0.700 \sim 1.185$ & 0.485 & 1.163 & $0.696 \sim 1.945$ & 0.565 \\
\hline \multicolumn{7}{|l|}{ GSE68465 (OS) } \\
\hline$H M M R$ & 1.001 & $1.000 \sim 1.002$ & 0.002 & 1.001 & $1.000 \sim 1.002$ & 0.045 \\
\hline gender & 1.427 & $1.101 \sim 1.849$ & 0.007 & 1.265 & $0.971 \sim 1.648$ & 0.081 \\
\hline age & 1.027 & $1.013 \sim 1.040$ & $<0.001$ & 1.029 & $1.015 \sim 1.043$ & $<0.001$ \\
\hline grade & 1.135 & $0.934 \sim 1.379$ & 0.204 & 0.960 & $0.769 \sim 1.199$ & 0.719 \\
\hline $\mathrm{N}$ classification & 2.012 & $1.712 \sim 2.365$ & $<0.001$ & 1.982 & $1.683 \sim 2.335$ & $<0.001$ \\
\hline $\mathrm{T}$ classification & 1.665 & $1.387 \sim 1.998$ & $<0.001$ & 1.423 & $1.174 \sim 1.725$ & $<0.001$ \\
\hline \multicolumn{7}{|l|}{ GSE68465 (PFS) } \\
\hline$H M M R$ & 1.002 & $1.001 \sim 1.003$ & 0.001 & 1.001 & $1.000 \sim 1.003$ & 0.012 \\
\hline gender & 1.224 & $0.872 \sim 1.718$ & 0.243 & 1.230 & $0.871 \sim 1.736$ & 0.240 \\
\hline age & 1.010 & $0.991 \sim 1.030$ & 0.305 & 1.017 & $0.998 \sim 1.037$ & 0.085 \\
\hline grade & 1.492 & $1.125 \sim 1.979$ & 0.006 & 1.208 & $0.892 \sim 1.634$ & 0.222 \\
\hline $\mathrm{N}$ classification & 1.559 & $1.257 \sim 1.934$ & $<0.001$ & 1.570 & $1.251 \sim 1.971$ & $<0.001$ \\
\hline $\mathrm{T}$ classification & 1.498 & $1.175 \sim 1.909$ & 0.001 & 1.253 & $0.961 \sim 1.635$ & 0.096 \\
\hline
\end{tabular}

2 Abbreviation: LUAD, lung adenocarcinoma; HR, hazard ratio; CI, confidence interval; $\mathrm{P}: p$ -

3 value; OS, overall survival; PFS, progression-free survival. 